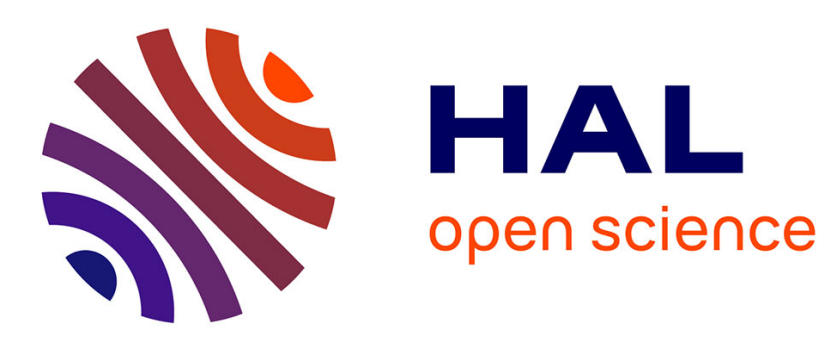

\title{
A new numerical methodology for simulation of unstable crack growth in time independent brittle materials
}

\author{
Bruno Michel, Thomas Helfer, Isabelle Ramière, Coralie Esnoul
}

\section{To cite this version:}

Bruno Michel, Thomas Helfer, Isabelle Ramière, Coralie Esnoul. A new numerical methodology for simulation of unstable crack growth in time independent brittle materials. Engineering Fracture Mechanics, 2018, 188, pp.126-150. 10.1016/j.engfracmech.2017.08.009 . cea-03581214

\section{HAL Id: cea-03581214 https://hal-cea.archives-ouvertes.fr/cea-03581214}

Submitted on 19 Feb 2022

HAL is a multi-disciplinary open access archive for the deposit and dissemination of scientific research documents, whether they are published or not. The documents may come from teaching and research institutions in France or abroad, or from public or private research centers.
L'archive ouverte pluridisciplinaire HAL, est destinée au dépôt et à la diffusion de documents scientifiques de niveau recherche, publiés ou non, émanant des établissements d'enseignement et de recherche français ou étrangers, des laboratoires publics ou privés. 


\title{
A new numerical methodology for simulation of unstable crack growth in time independent brittle materials
}

\author{
B. MICHEL*, T. HELFER*, I. RAMIERE*, C. ESNOUL** \\ * CEA, DEN, DEC, SESC, LSC bat 151 Centre de Cadarache 13108 Saint Paul Lez Durance FRANCE \\ ** EDF R\&D - Site des Renardières - Dpt MMC Avenue des Renardières, 77818 Moret sur Loing - France
}

\begin{abstract}
This paper focuses on a new algorithm for the quasi-static simulation of unstable crack propagation in time-independent brittle material. The proposed approach is based on a combination of the standard Newton's algorithm with a fictive path loading algorithm. First, a time step refinement process, associated to the Newton's algorithm, enables us to efficiently detect the beginning of unstable crack extension. Then, a fictive path loading algorithm is performed to assess an estimation of the post instability solution. Finally, this estimated solution is used as initial guess in the standard Newton's algorithm for the same time increment. The final converged solution satisfies both the mechanical equilibrium and the mechanical behaviour integration. This paper highlights several advantages of the proposed algorithm such as robustness, easy implementation on an existing Newton's type solver, genericity for different unstable nonlinear physical problems. An application of this new method is presented for a smeared crack model, based on a local continuous damage formulation, devoted to the simulation of brittle nuclear fuel rupture. The efficiency of the proposed algorithm is demonstrated through a set of $2 \mathrm{~d}$ and $3 \mathrm{~d}$ simulation results.
\end{abstract}




\begin{tabular}{|c|c|}
\hline \multicolumn{2}{|l|}{ Nomenclature } \\
\hline Latin symbols & \\
\hline$e_{\mathrm{r}}, e_{a}$ & Relative and absolute error given for the stress residual of the static equilibrium \\
\hline$\underline{\underline{E}}$ & Fourth order tensor of the elastic behaviour (Hooke law) \\
\hline$[E]$ & Finite element stiffness associated to the material elastic behaviour \\
\hline $\mathcal{L}$ & Incremental Lagrange function \\
\hline $\mathrm{G}_{x}$ & Nonlinear function derived from the minimisation of $\mathcal{L}$ with respect to $x$ \\
\hline$g$ & Damage law for the brittle rupture \\
\hline $\mathrm{G}_{\mathrm{c}}$ & Dissipated energy per unit surface of crack \\
\hline$H_{f}$ & Softening modulus of a local damage law \\
\hline$K, R$ & Tangent operator and residual of the Newton formulation \\
\hline $\overrightarrow{\mathrm{n}}$ & External normal to the surface \\
\hline$\overline{\bar{n}}_{i}$ & Unit tensor associated to the crack basis \\
\hline$\vec{u}$ & Displacement vector field according a Lagrangian formulation \\
\hline $\overrightarrow{\mathrm{u}}^{*}$ & Weak formulation test function \\
\hline$V_{R}, S_{R}$ & Volume of the damage zone and associated surface \\
\hline$\vec{w}_{i}$ & Unit vectors used to define an orthonormal crack basis \\
\hline \multicolumn{2}{|l|}{ Greek symbols } \\
\hline$\alpha$ & Internal state variable for nonlinear material constitutive equations \\
\hline$\Delta x$ & Time increment of the variable $x$ \\
\hline$\delta x$ & Iterative variation of $x$ during the Newton algorithm \\
\hline$\overline{\bar{\varepsilon}}$ & Strain tensor according a small strain assumption \\
\hline$\overline{\bar{\varepsilon}}^{*}$ & Strain tensor associated to the weak formulation test function $\overrightarrow{\mathrm{u}}^{*}$ \\
\hline$\overline{\bar{\varepsilon}}^{\text {tot }}, \overline{\bar{\varepsilon}}^{\text {crack }}$ & Total strain and mean crack strain associated to the displacement discontinuity \\
\hline$\overline{\bar{\sigma}}$ & Cauchy stress tensor \\
\hline$\sigma_{R}$ & Rupture stress \\
\hline
\end{tabular}

\section{INTRODUCTION}

Numerical simulation of crack initiation and growth in nuclear fuel ceramics is an important issue to assess mechanical integrity of the first confinement barrier for radio elements. Under irradiation, fuel pellet macroscopic fragmentation has to be considered because of its impact on the cladding loading and associated risk of cladding rupture [1]. Pellet fragmentation at microscopic scale is also an important issue for Loss Of Coolant Accident (LOCA) transient loading condition [2]. Since years 2000 this has 
been studying in our laboratory [3], [4] in the framework of a cooperative program between EDF, AREVA and the CEA. These studies concern Pellet Cladding Interaction (PCI) phenomenon in fuel rods of Pressurized Water Reactors (PWRs), in order to take into account the impact of pellet fragmentation in Stress Corrosion Cracking cladding rupture [6], and also fuel dispersal risk assessment under a LOCA [2]. A first smeared crack model [3] has been proposed and implemented in the fuel performance software environment PLEIADES [1]. This approach is consistent with the Griffith theory and has been used for many years to simulate stable crack extension in the 1D, 2D and 3D fuel models of the PWR fuel performance code ALCYONE [7] of the PLEIADES platform. However, in this approach unstable crack extension simulation is still a limitation of our quasi static nonlinear finite element solver. This topic has to be addressed in other application fields than nuclear fuel as far as we have to deal with a brittle material. The objective of this paper is then to propose a new numerical methodology in order to be able to extend the quasi static approach to the simulation of unstable crack extension.

In a first part, the new numerical approach for unstable crack extension is presented with the bibliographic background. Then, an updated version of the continuous damage model used to simulate brittle rupture in nuclear fuels is presented. In the last part, simulation results, based on the new numerical approach and the continuous damage model, are discussed with a set of 2D and 3D applications.

\section{UNSTABLE RUPTURE SIMULATION WITH A QUASI STATIC APPROACH}

A first approach to deal with unstable rupture is to switch to a truly dynamic simulation of the crack propagation (Teng [14], Acary [15]), the description of which is out of the scope of this paper, in order to compute kinetic energy induced by the difference between dissipation rate and elastic energy restitution rate. However the numerical integration associated to this approach is a real challenge, due to the very small time steps required to catch stress waves associated to the kinetic energy increase in the structure, and due to the computation of the damping effects linked to material dissipation or non-reflective stress wave boundary conditions. Although the issue of small time steps can be overcome using classical mass scaling techniques (Bobineau, Maurel, and Morançay [16]), this dynamic approach is not considered here as the time steps required are not tractable for our applications. It is however the idea of equivalent dynamic steps which is the basis of the algorithm presented here.

\subsection{Quasi-static formulation}

Expressing the problem of crack evolution as a minimization problem is the basis of the pioneering work of Francfort and Marigo [8], Bourdin, Francfort, and Marigo [9] or Amor, Marigo, and Maurini [10]. For standard generalized materials (Halphen and Nguyen [11]) the evolution of a mechanical system in quasistatic loading can be expressed as a minimum problem of an incremental Lagrange function $\mathcal{L}$ (See Germain, Nguyen, and Suquet [12], Lorentz and Andrieux [13] for details) with respect to the displacement increment $\Delta \vec{u}$ and the state variables increment $\Delta \alpha$ : 


$$
(\Delta \vec{u}, \Delta \alpha)=\underset{\Delta \vec{v}, \Delta \beta}{\operatorname{argmin}} \mathcal{L}\left(\Delta \vec{v}, \Delta \beta,\left.\vec{u}\right|_{t},\left.\alpha\right|_{t}, d t\right)
$$

This framework is used in this introduction to simplify the discussion but this does not entail the generality of the conclusions that will be drawn here. For the sake of readability, variables describing the state of the system at the beginning of the time step $\left(\left.\vec{u}\right|_{t},\left.\alpha\right|_{t}, d t\right)$ are omitted in the following. The Gâteaux derivative of the Lagrange function $\mathcal{L}$ with respect to the displacement increment is equivalent to the virtual work principle (2) :

$$
\forall \vec{u}^{\star} \int_{V} \overline{\bar{\sigma}}(\overline{\bar{\varepsilon}}(\Delta \vec{u}), \Delta \alpha): \overline{\bar{\varepsilon}}^{*} \cdot d V=\int_{S} \vec{T} \cdot \vec{u}^{*} d S
$$

Where $\overline{\bar{\sigma}}$ is the Cauchy stress tensor, $\overline{\bar{\varepsilon}}$ the strain, $\vec{T}$ the prescribed traction on the boundary $S, \vec{u}^{*}$ is a test function (or virtual displacement) and $\bar{\varepsilon}^{*}$ its associated strain tensor (or virtual strain).

In the following, Equation (1) is replaced by its discrete equivalent obtained by a spatial discretisation based on the finite element method with a solution basis in an approximate finite dimension space. Again, for the sake of simplicity, the same notations are used for the discrete approximation of the unknowns $\Delta \vec{u}$ and $\Delta \alpha$. To solve the discrete formulation of Equation (1) various approaches have been proposed in the literature, most of them are based on an iterative algorithm. Minimization with respect to $\Delta \vec{u}$ and $\Delta \alpha$ can often be decoupled and this leads to use standard fixed point algorithms. For example, let $\Delta \vec{u}^{(i)}$ and $\Delta \alpha^{(i)}$ be the current estimation of the displacement field increment and the internal state variable increment. A new estimation of the internal state variable increment $\Delta \alpha^{(i+1)}$ is obtained thanks to function $G_{\alpha}$ describing the mechanical behaviour (3). The latter is equivalent to a minimization step with respect to $\Delta \alpha$ as it can be demonstrated for given $G_{\alpha}$.

$$
\Delta \alpha^{(i+1)}=G_{\alpha}\left(\Delta \vec{u}^{(i)}\right)
$$

For local behaviours, $G_{\alpha}$ is the mechanical behaviour integration step, performed at each integration point: in this case, expression of $G_{\alpha}$ is based on the strain increment. For phase-field or non-local behaviours, various operators can be used.

In the most common algorithms, an update value of the stress tensor, associated to $\Delta \alpha^{(i+1)}$, is then used to get a new estimation of the displacement increment $\Delta \vec{u}^{(i+1)}$ via the residual $\mathrm{R}_{\vec{u}}$ derived from equation (2). This correction often takes the form of (4) given by the Newton's like algorithm where $K$ is an appropriate search operator.

$$
\Delta \vec{u}^{(i+1)}=G_{\vec{u}}\left(\Delta \vec{u}^{(i)}, \Delta \alpha^{(i+1)}\right)=\Delta \vec{u}^{(i)}-K^{-1} R_{\vec{u}}\left(\Delta \vec{u}^{(i)}, \Delta \alpha^{(i+1)}\right)
$$

\subsection{Unstable crack extension simulation}

\subsubsection{Convergence of the Newton algorithm}

To discuss this point a schematic illustration is given in Figure 1. This example shows that in case of unstable crack extension the solution of equation (2) is not possible without a reverse displacement under 
unstable crack propagation (black curve between point A and B in Figure 1) when the softening behaviour leads to internal forces smaller than the internal force induced by the elastic energy restitution rate under a constant displacement boundary condition (red line $\mathrm{AB}$ in Figure 1). For the standard iterative Newton algorithm, the tangent operator will lead to predict an increasing displacement increment after point $\mathrm{A}$, which means that the residual $R_{\vec{u}}$ will suddenly increase with a jump of the solution beyond point $\mathrm{B}$ in Figure 1. This jump will be characterized by a discontinuous evolution of the displacements $\Delta \vec{u}$ and the state variables $\Delta \alpha$ that would not tend toward zero as the time step diminishes. This discontinuous evolution of the state variables is associated with a loss of energy in the system: physically, this energy is transformed in kinetic energy which is not taken into account in a quasi-static approach. After unstable crack propagation (beyond B in Figure 1) the structure reaches a new static equilibrium state, but the details of this transition cannot be described. Indeed, there are dynamical phenomena that can't be captured by a quasi-static approach: eigen vibration modes to compute local inertial energy, interaction of the crack path with elastic waves reflected on the body boundary, saw-tooth crack path at the microscopic level which dissipates more energy than straight propagation, etc... The physical validity of the solution found by a quasi-static approach thus greatly depends on the case treated. From a numerical point of view, the equilibrium point at the onset of the unstable crack propagation is a saddle point of the Lagrange function, i.e. is characterized by the loss of convexity of the problem and a tangent operator no more definite positive. The last point and the lack of static solution in the vicinity of the previous solution explain most of the numerical difficulties reported in the literature. Thus, an important issue for unstable crack simulation concerns the choice of the numerical algorithm to solve the partial derivative equations system (2).

\subsubsection{Globalization of the standard Newton algorithm}

As the minimum problem associated to the mechanical equilibrium locally loses its convexity, the standard Newton algorithm, Equation (4) is no more appropriate. To overcome this problem many authors propose to combine the Newton algorithm with a globalization technique. Among the large number of methods proposed in the literature, the trust region Newton method or the Powell DogLeg method are well-known approaches. In computational mechanics, the most popular approach however relies on combining the Newton algorithm with a line-search technique (See Belytschko [17] and the references therein).

\subsubsection{Unbalanced steps}

Another solution consists in accepting solutions that do not satisfy the mechanical equilibrium at intermediate time steps. The Implex algorithm by Oliver and al. [18] extends this idea. In this case, it is important to note that the displacements are corrected using extrapolated values of the state variables $\Delta \tilde{\alpha}$ 
from the previous steps (5). Once $\Delta \vec{u}$ is known, the evolution of the state variables is given by Equation (6).

$$
\begin{aligned}
\Delta \vec{u} & =G_{\vec{u}}(\overrightarrow{0}, \Delta \tilde{\alpha}) \\
\Delta \alpha & =G_{\alpha}(\Delta \vec{u})
\end{aligned}
$$

An adaptive time step strategy using a predictor/corrector scheme is introduced to control the error. As discussed before, the time-continuity of the displacement and of the state variables cannot be assumed for unstable crack propagation. Thus, this class of algorithms is not directly suited for our needs. More recently, in the context of phase field modelling of the crack propagation, Miehe [19] proposed to accept unbalanced steps where the damage results, obtained from the values of the elastic energies at the beginning of the time step (7), and the displacements are only corrected using the secant tangent operator (8) (See Miehe, Hofacker, and Welschinger [19] and Nguyen et al. [20] for details). Nguyen et al. [21] improved this algorithm by converging on the displacements at constant damage over the time step (9), leading to the following algorithm.

$$
\begin{aligned}
\Delta \alpha & =G_{\alpha}(\Delta \overrightarrow{0}) \\
\Delta \vec{u} & =\operatorname{argmin}_{\Delta \vec{v}} \mathcal{L}(\Delta \vec{v}, \Delta \alpha)
\end{aligned}
$$

Equation (8) is solved as following :

$$
\Delta \vec{u}^{(i+1)}=G_{\vec{u}}\left(\Delta \vec{u}^{(i)}, \Delta \alpha\right)
$$

\subsubsection{Control loading path}

Controlled loading path algorithms [27] introduce a modification of the loading time history in order to make the damage stage consistent with the static equilibrium during the unstable rupture and lead to a mechanical response presenting snap-backs as illustrated in Figure 1. Such algorithms encompass the classical arc-length methods which introduce a loading control parameter $\Delta \tau$. The latter is a new unknown of the problem and a local control loading criterion is added to the nonlinear mechanical formulation in order to obtain a well-posed problem. If we refer to the results presented in reference [27], this new algorithm leads to a lot of iterations associated to a very complex loading time history during the damage stage. Moreover, to avoid elastic unloading a special care is brought to the control loading criterion.

\subsection{Main features of the Cast3M algorithm}

The Cast3M algorithm used to solve the nonlinear equilibrium equation (2) is described in Figure 2. Compare to a standard Newton algorithm the following modification have been proposed:

- the Jacobian matrix is replaced by a stiffness matrix derived from the undamaged material elastic behaviour: it leads to a so-called quasi Newton Algorithm (QNA),

- a fixed point acceleration method based on the equilibrium residual nodal forces is used, 
- a Fictive Path Loading Algorithm (FPLA) is used to overcome convergence problems.

The QNA is based on a fixed point method using equations (10) and (11).

$$
\begin{aligned}
& {[E] .\{\delta \Delta U\}^{i}=\left\{R_{\vec{u}}\right\}_{t+\Delta t}^{i-1}} \\
& \left\{\begin{array}{c}
\sigma \\
\alpha
\end{array}\right\}_{t+\Delta t}^{i}=\mathrm{F}\left(\left\{\begin{array}{c}
\sigma \\
\alpha
\end{array}\right\}_{t},\{\Delta U\}^{i}, d t\right)
\end{aligned}
$$

Where $[E]$ is the undamaged stiffness matrix, $\delta$ is the iterative variation of the solution, $\{\Delta U\}^{i}$ is the nodal displacement increment between $t$ and $t+\Delta t$ at iteration $i,\{R\}_{t+\Delta t}^{i-1}$ is the nodal equilibrium residual, $\sigma$ and $\alpha$ are respectively the stresses and the material internal state variable defined at the Gauss integration points and $\mathrm{F}$ is the nonlinear material law. Equation (10) returns to Equation (4) with $G_{\vec{u}}=$ $\{\Delta U\}-[E]^{-1} \cdot\left\{R_{\vec{u}}\right\}$.

The undamaged stiffness matrix $[E]$ can be easily computed compared to the consistent tangent operator and has nice mathematical properties (symmetrical, positive definite,etc.). Moreover in case of softening behaviour a local material stiffness equals to zero doesn't lead to a singular matrix in this QNA.

The nodal equilibrium residual is derived from equation (12), where $\left\{F_{\text {ext }}\right\}$ is the nodal external loading, right hand side of equation (2), and $[B]$ is the strain-displacement matrix.

$$
\left\{R_{\vec{u}}\right\}=\left\{F_{\text {ext }}\right\}-\int_{V}[B] \cdot\{\sigma\} \cdot d V
$$

However, such a quasi-Newton algorithm is of first-order and may converge very slowly. So, in order to increase the convergence rate, a fixed point acceleration method based on the equilibrium residual nodal forces is used. This method, presented in reference [30], is an Anderson method [31] summarized by the following equations:

$$
\begin{aligned}
& \Delta \vec{u}^{(i+1)}=G_{\vec{u}}\left(\Delta \vec{u}^{(i)}\right)-\lambda_{1}^{(i)}\left(G_{\vec{u}}\left(\Delta \vec{u}^{(i)}\right)-G_{\vec{u}}\left(\Delta \vec{u}^{(i-1)}\right)\right)-\lambda_{2}^{(i)}\left(G_{\vec{u}}\left(\Delta \vec{u}^{(i)}\right)-G_{\vec{u}}\left(\Delta \vec{u}^{(i-2)}\right)\right) \\
&-\lambda_{3}^{(i)}\left(G_{\vec{u}}\left(\Delta \vec{u}^{(i)}\right)-G_{\vec{u}}\left(\Delta \vec{u}^{(i-3)}\right)\right)
\end{aligned}
$$

The coefficients $\lambda_{1}^{(i)}, \lambda_{2}^{(i)}$ and $\lambda_{3}^{(i)}$ are obtained by quadratically minimizing the Euclidean norm of

$$
\begin{gathered}
R_{\vec{u}}\left(\Delta \vec{u}^{(i)}\right)-\lambda_{1}^{(i)}\left(R_{\vec{u}}\left(\Delta \vec{u}^{(i)}\right)-R_{\vec{u}}\left(\Delta \vec{u}^{(i-1)}\right)\right)-\lambda_{2}^{(i)}\left(R_{\vec{u}}\left(\Delta \vec{u}^{(i)}\right)-R_{\vec{u}}\left(\Delta \vec{u}^{(i-2)}\right)\right) \\
-\lambda_{3}^{(i)}\left(R_{\vec{u}}\left(\Delta \vec{u}^{(i)}\right)-R_{\vec{u}}\left(\Delta \vec{u}^{(i-3)}\right)\right)
\end{gathered}
$$

In order the accelerated displacement to respect the boundary conditions, the Cast3M software works on the corresponding accelerated residual:

$R_{\vec{u}}^{a c c}\left(\Delta \vec{u}^{(i)}\right)=\left(\lambda_{1}^{(i)}+\lambda_{2}^{(i)}+\lambda_{3}^{(i)}\right) R_{\vec{u}}^{*}\left(\Delta \vec{u}^{(i)}\right)+\left(\lambda_{2}^{(i)}+\lambda_{3}^{(i)}\right) R_{\vec{u}}^{*}\left(\Delta \vec{u}^{(i-1)}\right)+\lambda_{3}^{(i)} R_{\vec{u}}^{*}\left(\Delta \vec{u}^{(i-2)}\right)$

where $R_{\vec{u}}^{*}\left(\Delta \vec{u}^{(i)}\right) \stackrel{\text { def }}{=}[E]\left(G_{\vec{u}}\left(\Delta \vec{u}^{(i)}\right)-G_{\vec{u}}\left(\Delta \vec{u}^{(i-1)}\right)\right)$

As the search operator is non-iteration dependant, substitution of Eq. (15) in Eq (13) gives

$\Delta \vec{u}^{(i+1)}=G_{\vec{u}}\left(\Delta \vec{u}^{(i)}\right)-[E]^{-1} R \underset{\vec{u}}{a c c}\left(\Delta \vec{u}^{(i)}\right)$ 
Hence, the fixed point iteration (10) is modified as

$[E] .\{\delta \Delta U\}^{i+1}=\left\{R_{\vec{u}}\right\}_{t+\Delta t}^{i}-\left\{R^{a c c}\right\}_{t+\Delta t}^{i}$

Finally $R_{\vec{u}}^{*}$ can also be expressed as

$R_{\vec{u}}^{*}\left(\Delta \vec{u}^{(i)}\right)=R_{\vec{u}}\left(\Delta \vec{u}^{(i)}\right)-R_{\vec{u}}^{a c c}\left(\Delta \vec{u}^{(i-1)}\right)$

In practice, the Cast $3 \mathrm{M}$ acceleration algorithm (17) is applied after the third iteration of the fixed point method (10) and then every other iteration.

The main idea of the FPLA is to do virtual steps when a non-convergence of the Newton algorithm is detected. A maximal number $f$ of iterations is given to reach the convergence, even for the virtual steps. Hence, after the $f$ first QNA iterations, the FPLA is activated. The integer (if $p-1)$ represents the virtuel steps counter. When the FPLA is activated the initial state at time $t$ is replaced by the stresses and the state variables associated to the intermediate non equilibrated solution at iteration $i=$ if $p \times f$ and the total displacement increment is reinitialized to zero (see virtual loading path loop in Figure 2). As a consequence of this virtual loading path, once the FPLA is activated the time step is characterized by a null time increment and the system evolves only to equilibrate the structure. This null time step has to be taken into account in the integration of the nonlinear behaviour (11). This can be done without modification in an implicit integration or with a minimal time step, avoiding time dependent behaviour, in an explicit integration. The FPLA converge when the solution reaches the equilibrium given by the residual nodal forces.

In our studies a new convergence criterion has been proposed (18) checking the convergence of the stress tensor at each Gauss point. This convergence criterion is compatible with the proposed QNA as it lies on the same fourth order elastic tensor than the approximate Jacobian matrix, see Equation (19). In order to have an efficient criterion even in case of inelastic stress stationarity, this criterion is based on the difference between an extended elastic stress solution and the non-linear stress tensor derived from the behaviour integration. Moreover, this proposed criterion turns automatically from a relative error to an absolute error, respectively $e_{r}$ and $e_{a}$ in Equation (18), when the stress values are small.

$$
\begin{aligned}
& \left.\max \left(\left\{R_{\overline{\bar{\sigma}}}\right\}^{i}-e_{r} \cdot\{\overline{\bar{\sigma}}\}_{t+\Delta t}^{i}\right)\right|_{\text {Gauss Point }}<e_{a} \\
& \left\{R_{\overline{\bar{\sigma}}}\right\}^{i}=\{\overline{\bar{\sigma}}\}_{t+\Delta t}^{i}-\left(\{\overline{\bar{\sigma}}\}_{t+\Delta t}^{i-1}+\left\{\underline{\left.\left.\underline{E}: \overline{\bar{\varepsilon}}\left(\delta \Delta U^{i}\right)\right\}\right)}\right.\right.
\end{aligned}
$$

\subsection{New methodology for unstable rupture simulation}

The new approach proposed in this paper is based on a three steps method as following:

- a time step refinement algorithm to detect the beginning of instable crack extension,

- a FPLA to assess the post instability solution,

- a QNA initialized with the FPLA's solution to validate the results. 
The objective of the time step refinement method is to start the unstable crack extension stage as close as possible of the crack initiation loading. To this end we make the assumption that, except in case of unstable crack extension, it is always possible to make the QNA converge by reducing the time step increment. Based on the previous assumption a persistent non-convergence of the QNA when the time step tends toward zero is due to an unstable crack extension. Hence, the beginning of the unstable rupture is numerically detected thanks to a small enough time step threshold. From a practical point of view the minimal time step threshold, used for the detection of the unstable crack propagation, is defined in order to be consistent with a small variation of the prescribed loading. This guarantees that the last converged time step is close enough to the unstable crack extension loading. Nevertheless if this time step is not small enough and hence the non-convergence is not due to an unstable crack extension, the probably invalid FPLA's solution would be detected by the third step of the proposed method.

In case of uniform stress fields the QNA can find a uniform damage solution because the convergence precision can lead to miss the damage localization at crack initiation. To overcome this problem the time step is also refined if the number of damaged element during a time step is greater than a half of the total number of element.

In the second step of the proposed method the unstable crack solution is computed with the FPLA described in section 2.3. In our case, where a dynamic equilibrium is involved during instability, one can consider that the residual of unbalanced virtual steps is equivalent to the dynamic forces induced by the kinetic energy. The main idea lies on the fact that the solution reached after the unstable crack extension will return consistent with a static equilibrium if the external loading is based on Dirichlet boundary conditions. From the fully dynamic approach point of view, it means that the post-instability static solution is consistent with a state where the kinetic energy induced during the damage process vanishes because of material dumping and energy transport. From the path following constraint method point of view, the proposed approach returns to the case where the reverse loading is no more required to respect the static equilibrium (stages II in Figure 1).

To confirm the validity of the virtual loading steps solution, the unstable step is recomputed using the fictive path loading solution as estimation of the solution in the Cast3M QNA without FPLA option. In this case, an initial value of nodal displacement $\left(\{\Delta U\}^{i n i}\right.$ in Figure 2) is given. If this solution is valid, the convergence must be reached in very few iterations: in this case the new solution both satisfies the equilibrium and the mechanical behavior according to a unique displacement increment at the end of the time step.

The fact that virtual steps are characterized by a null time increment makes this procedure mostly suitable for time-independent materials, as the time dependent part will be associated to a non-equilibrated solution. However, for behaviors combining time-dependent and time-independent mechanisms, the last step of the algorithm (initialization of the QNA) will correct the virtual step solution. The main interests of this algorithm are: 
- $\quad$ its robustness, as demonstrated by the various examples provided in this paper;

- $\quad$ its easy implementation, as it only consists in a loop over the standard Newton algorithm and a final restart of the Newton algorithm with a prescribed initial guess;

- $\quad$ its genericity, as it can be used with any kind of instability (unstable crack propagation, buckling) in small and finite strain, without any modification to the mechanical behavior. The restriction to timeindependent mechanism has been discussed earlier

- $\quad$ it provides a solution that satisfy the equilibrium equation and the mechanical behaviour.

\section{CONTINOUS DAMAGE APPROACH OF THE RUPTURE PROCESS}

\subsection{Smeared crack model formulation for nuclear fuels}

The formulation of the smeared crack model is given by Equations (20) to (22).

$$
\begin{aligned}
& \overline{\bar{\sigma}}=\underline{\underline{E}}:\left(\overline{\bar{\varepsilon}}^{t o t}-\overline{\bar{\varepsilon}}^{\text {crack }}\right) \\
& \overline{\bar{\varepsilon}}^{\text {crack }}=\sum_{i} \varepsilon_{i}^{\text {crack }} \cdot \vec{w}_{i} \otimes \vec{w}_{i} \\
& g\left(\overline{\bar{\sigma}}:\left(\vec{w}_{i} \otimes \vec{w}_{i}\right), \varepsilon_{i}^{\text {crack }}\right)=0
\end{aligned}
$$

Where $\underline{\underline{E}}$ is the fourth order elastic tensor, $\overline{\bar{\varepsilon}}^{\text {crack }}$ is the crack strain tensor, $\vec{w}_{i}$ is the unit vectors used to define an orthonormal crack basis and $g$ is the damage law for brittle rupture.

Material softening during the damage process is given by the linear function $g$ illustrated in Figure 3 . In the latter stage 2 represents the damage process from microscopic cracks up to a macroscopic rupture where the material stiffness is equal to zero. Crack closure due to unloading is also possible thanks to the model (stages 6 and 5 in Figure 3). If unloading occurs during the softening stage, the unloading path is assumed to be elastic with an affine linear stiffness. It means that local plasticity due to microscopic cracks is negligible, which seems reasonable for brittle rupture. After a complete crack closure the material can recover its elastic stiffness (stage 7 in Figure 3) under a compressive stress state defined in the fracture basis. In this paper the fracture basis is derived from the stress tensor's eigenvalues at crack initiation. At this stage we didn't introduce constitutive equations to model material softening under a shear strain loading occurring in the fracture basis after crack initiation. Moreover, in this formulation it is assumed that the stress eigen basis doesn't change during the softening process (radial stress relaxation). For the numerical resolution of the smeared crack model with an implicit method, the constitutive equations of the function $g$ are defined in Table 1. The damage law $g$ is a piecewise linear function depending on the stage considered in Figure 3. In order to select which stage is consistent with the iterative solution of the implicit solver, a set of required conditions has been defined as given in Table 1. 
Then the Jacobian matrix and the residual of the Newton method are computed using the appropriate definition of $g$.

\begin{tabular}{|c|c|c|}
\hline $\begin{array}{c}\text { Stage } \\
\text { (see Figure 3) }\end{array}$ & $\begin{array}{l}\text { Required condition to select the } \\
\text { damage stage }\end{array}$ & Damage law equation $g\left(\overline{\bar{\sigma}}: \overline{\bar{n}}_{\mathrm{i}}, \varepsilon_{i}^{\text {crack }}\right)=0$ \\
\hline 1 & $\left.\varepsilon_{i}^{\text {crack }}\right|_{\max (t)}=0$ and $\sigma_{1}^{p}<\sigma_{R}$ & $\varepsilon_{i}^{\text {crack }}=0$ \\
\hline 2 & $\begin{array}{c}0<\left.\varepsilon_{i}^{\text {crack }}\right|_{\max (t)}<\frac{\sigma_{R}}{H_{f}} \\
\text { and } \\
\varepsilon_{i}^{\text {crack }}=\left.\varepsilon_{i}^{\text {crack }}\right|_{\max (t)}\end{array}$ & $\overline{\bar{\sigma}}: \bar{n}_{i}-\left(\sigma_{R}-H_{f} \cdot \varepsilon_{i}^{c r a c k}\right)=0$ \\
\hline $3-6$ & $\left.\varepsilon_{i}^{\text {crack }}\right|_{\max (t)}>\frac{\sigma_{R}}{H_{f}}$ and $\varepsilon_{i}^{\text {crack }}>0$ & $\overline{\bar{\sigma}}: \bar{n}_{i}=0$ \\
\hline $4-5$ & $\begin{array}{c}0<\left.\varepsilon_{i}^{\text {crack }}\right|_{\max (t)}<\frac{\sigma_{R}}{H_{f}} \\
\text { and } \\
0<\varepsilon_{i}^{\text {crack }}<\left.\varepsilon_{i}^{\text {crack }}\right|_{\max (t)}\end{array}$ & $\bar{\sigma}:=n_{i}-\left(\frac{\sigma_{R}}{\left.\varepsilon_{i}^{\text {crack }}\right|_{\max (t)}}-H_{f}\right) \cdot \varepsilon_{i}^{\text {crack }}=0$ \\
\hline 7 & $\left.\varepsilon_{i}^{\text {crack }}\right|_{\max (t)}>0$ and $\varepsilon_{i}^{\text {crack }}=0$ & $\varepsilon_{i}^{\text {crack }}=0$ \\
\hline
\end{tabular}

Table 1: Analytical expressions of the damage law $\left(\overline{\bar{n}}_{i}=\vec{w}_{i} \otimes \vec{w}_{i},\left.\varepsilon_{i}^{\text {crack }}\right|_{\max (t)}\right.$ is the historical maximal value of the cracking strain in direction $i, \sigma_{1}^{p}$ is the maximal principal stress)

As defined in Table 1 the damage law $g$ has two material parameters $\sigma_{R}$ and $H_{f}$ discussed in the next section.

\subsection{Physical meaning of the rupture parameters}

\subsubsection{Softening modulus and mesh size effect}

Considering the rupture process of a brittle material, as illustrated with stage 2 in Figure 3, the continuum damage approach has to deal with material damage initiation, material softening and free surface creation at the continuum material scale description. In a brittle material one considers [25] that material softening is the result of micro-cracks ${ }^{1}$ propagation up to a macroscopic size regarding the continuum description.

${ }^{1}$ Micro has to be considered as a subscale of the continuum media scale representation. 
Based on this consideration it appears that the energy requested for macroscopic crack nucleation is related to the material toughness. In order to ensure the consistency of the total energy dissipated in the continuous damage approach of section 3.1, balance equation (23) is used.

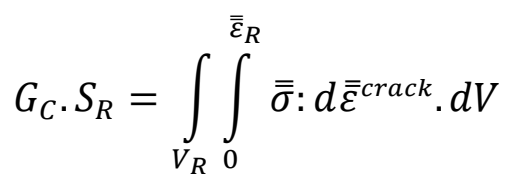

Where $G_{C}$ is the dissipated energy per surface unit, $\overline{\bar{\sigma}}$ is the Cauchy stress tensor, $\overline{\bar{\varepsilon}}^{\text {crack }}$ is the local strain induced by the damage process, $S_{R}$ is the cracked surface, $\overline{\bar{\varepsilon}}_{R}$ is the rupture strain at macroscopic crack nucleation and $V_{R}$ is the volume of the damage zone.

If we introduce the definition of the damage function $g$ during stage 2 in equation (23) we can compute the softening modulus as following:

$$
H_{f}=\frac{\sigma_{R}^{2}}{2 . G_{C}} \cdot \frac{V_{R}}{S_{R}}
$$

Where $\sigma_{R}$ is the rupture stress of the damage law.

Equation (24) gives the regularization method required to ensure that the dissipated energy will not be mesh size dependent in the finite element computation. The characteristic length defined as the ratio $V_{R} / S_{R}$ of the finite element can be computed for each crack orientation as the scalar product between the mesh size vector and the unit vector normal to the surface crack (see Eq. (25)).

$$
H_{f_{i}}=\frac{\sigma_{R}^{2}}{2 \cdot G_{C}} \cdot\left|\vec{h} \cdot \vec{w}_{i}\right|
$$

From a practical point of view the vector $\vec{h}$ is defined with the three edges of a cubic volume element equivalent to the real finite element (see Figure 4). In the case of an octahedral finite element the equivalent cubic element can be easily chosen, however for other finite element shape the definition of the mesh size vector $\vec{h}$ must be adapted. For our application on tetrahedral finite elements, a scalar mesh size parameter has been used with the direct computation of the ratio $V_{R} / S_{R}$ of the finite element.

Regarding Equations (24) or (25) the direct physical meaning of the characteristic length is not obvious, because it will depend on the local damage law formulation. In all cases the characteristic length is the size of the zone affected by the rupture process in the computation. The computational size will be consistent with the real damaged zone size only if the dissipated strain energy density is consistent with the one involved in the damage process. As the real size of the damaged zone can be very small, the characteristic length is often much greater in order to have an equivalent size consistent with the representative volume needed for a homogenized description of the strain and stress fields.

Thanks to this local softening modulus (23) we can avoid first order mesh size effect in our approach, at least to be consistent with a Griffith criterion in the case of an initial flaw [3], however some limitations 
still remain. A first limitation is due to the computation of the characteristic length which is based on an assumption of a uniform cracking strain in the finite element, and then can be easily transpose only on linear finite element. Another limitation is due to the crack path sensitivity to the mesh texture. In the case of mixed mode propagation and crack bifurcation, the local approach reduces the possible paths for propagation from one gauss point to another and leads to high sensitivity of the solution to the chosen finite element and the mesh organization with its apparent texture (see for instance mixed mode loading application in section 4.2). Despite these limitations this approach gives already very interesting results for applications where crack propagation is mainly under mode I loading and if we don't need too much precision in the crack path assessment.

\subsubsection{Rupture stress}

The second parameter of this continuous damage model is the rupture stress $\sigma_{R}$ which is usually defined as the experimental rupture stress observed in a rupture test without initial macroscopic crack. The physical meaning of this rupture stress should be clarified in order to understand the link between damage process at microscopic scale and macroscopic scale and to avoid inappropriate scale transfer. The rupture stress controls the beginning of the softening stage when the softening modulus controls the energy needed per surface unit to create the macroscopic crack according to the Griffith criterion. As the beginning of the softening stage is related to microscopic crack initiation for a brittle material, we can also use a Griffith criterion to define the relevant scale associated to this parameter. Then the rupture stress parameter can be seen as the result of Equation (26) where $a$ is the microscopic crack size. This leads to conclude that the rupture stress is scale-dependent and that we should use an appropriate experimental measure of this parameter. When a relevant rupture test can't be carried out, a scale transfer coefficient can be derived from the statistical distribution of subscale cracks in the material.

$$
\sigma_{R} \approx \sqrt{\frac{E \cdot G_{C}}{\pi \cdot a}}
$$

\section{SIMULATION RESULTS}

In order to test the capacity of the proposed algorithm to detect if crack propagation is stable or unstable different configurations have been tested in the following sections. Another objective of these tests under stable crack growth conditions is also to confirm that the convergence of the QNA can be obtained with the time step refinement, and that the FPLA is needed only for unstable crack growth steps. 


\subsection{Mode I loading in smooth and cracked specimens}

In order to simulate stable crack propagation for 2D configurations given in Figure 5, numerical values of Table 2 have been used.

\begin{tabular}{|c|c|c|c|c|c|c|c|c|}
\hline Specimen & $t$ & $L$ & $a$ & $E$ & $v$ & $G_{C}$ & $\sigma_{R}$ & $\varepsilon_{z z}^{i m p}$ \\
\hline Smooth & \multirow[t]{2}{*}{$0,25 \cdot 10^{-2}$} & \multirow[t]{2}{*}{$0,125 . \mathrm{t}$} & & \multirow[t]{2}{*}{$200 \mathrm{GPa}$} & \multirow[t]{2}{*}{0.3} & $50 \mathrm{~J} / \mathrm{m}^{2}$ & \multirow[t]{2}{*}{$164,7 \mathrm{MPa}$} & \multirow[t]{2}{*}{$0.13 \%$} \\
\hline Cracked 1 & & & $0,25 . t$ & & & $8 \mathrm{~J} / \mathrm{m}^{2}$ & & \\
\hline
\end{tabular}

Table 2 : Input data for 2D simulation of stable crack extension

Results for the smooth specimen are presented in Figure 6. As we can see, the stress-strain curve is consistent with a stable crack extension configuration because the slope of the curve in the softening stage is finite. This means that after crack initiation there is a static equilibrium where elastic energy restitution rate is equal to crack energy dissipation rate. Damage starts from the weakest element at the centre of the specimen, and then, when damage extension reaches approximately one fifth of the thickness (see Figure $6 \mathrm{~d}$ ), new cracks initiate at the bottom of the specimen because of the stress relaxation induced by damage extension at the middle of the specimen. The macroscopic stress-strain curve can be validated with analytical results of a cohesive zone model consistent with this one dimensional configuration. The stress level expected from the cohesive zone model, given in Figure 7, after a total strain of $0.13 \%$ is $95 \mathrm{MPa}$. This result is consistent with the smeared crack simulation (Figure 6) where the final stress is approximately $100 \mathrm{MPa}$ after a total strain of $0.13 \%$. As expected under stable crack growth condition, the FPLA is not necessary and the time step refinement method is enough to get the convergence during the crack growth stage.

To analyse the influence of the boundary condition on the damage extension, the same configuration has been tested with a homogenous stress boundary condition (HSBC) for the tensile loading. This type of boundary condition, proposed in [28], is defined with Equation (21). In the later the loading is given by the mean displacement and the HSBC is possible through the degrees of freedom given by the following linear equation.

$$
\frac{1}{\int_{S} d s} \cdot \sum_{i=1}^{n}\left(\int_{S} N_{i} \cdot u_{i}^{y} \cdot d s\right)-U_{i m p}^{y}=0
$$

$S$ is the surface with HSBC, $n$ the number of nodes of the surface $S, N_{i}$ the finite element shape functions, $u_{i}^{y}$ the vertical component of the nodal displacement and $U_{i m p}^{y}$ the mean displacement loading.

The damage extension at the end of the loading obtained with this boundary condition is given in Figure 8. When the local stress relaxation is not possible, there is only one crack initiated from the weakest element, and then the crack extension occurs in a single element row under a mode I loading. If we consider the crack length given by the smeared crack model and the stress level at the end of the loading, we can compute the energy restitution rate with the G_THETA method [29]. The numerical application, 
given in Figure 9, confirms that crack extension stops because the energy restitution rate is lower than material toughness set at $50 \mathrm{~J} / \mathrm{m}^{2}$. Just after the crack initiation there is a first stage where the crack extension is unstable, and then, when the crack size leads to enough stress relaxation, the crack extension is stable up to the end of loading (see these two stages in Figure 10). According these results we can see that the maximum load level is controlled by the rupture stress property and that the final crack size is controlled by the material toughness.

Results obtained for the cracked specimen are presented in Figure 11. The stress-strain curve shows a stable crack growth stage starting after the reaching of the maximum mean tensile stress. The stable crack growth is possible only with a non-uniform displacement boundary condition represented in Figure 5 for the cracked specimen 1. The elastic energy restitution rate ( $\mathrm{G}$ integral) is computed as a function of the displacement loading for different crack sizes defined through the smeared crack model damage results. As shown in Figure 11, the crack extension starts when the $G$ integral is greater than $8 \mathrm{~J} / \mathrm{m}^{2}$ and then during the stable crack, this integral almost constant around $9 \mathrm{~J} / \mathrm{m}^{2}$. This result confirms that the smeared crack model is consistent with a Griffith criterion. The sensitivity analysis, done in Figure 12, confirms that the crack extension doesn't depend on the mesh size and on the finite element as expected with the introduction of a characteristic length as discussed in section 3.2.1.

\begin{tabular}{|c|c|c|c|c|c|c|c|c|}
\hline Specimen & $t(\mathrm{~m})$ & $L$ & $a$ & $E$ & $v$ & $\overline{G_{C}}$ & $\sigma_{R}$ & $\varepsilon_{z z}^{i m p}$ \\
\hline Smooth & $0,25 \cdot 10^{-2}$ & \multirow[t]{2}{*}{$1,5 . \mathrm{t}$} & & \multirow[t]{2}{*}{$200 G P a$} & \multirow[t]{2}{*}{0.3} & $4 \mathrm{~J} / \mathrm{m}^{2}$ & \multirow[t]{2}{*}{$164,7 \mathrm{MPa}$} & \multirow[t]{2}{*}{$0.13 \%$} \\
\hline Cracked 2 & $0,125 \cdot 10^{-2}$ & & $0,5 . \mathrm{t}$ & & & $8 \mathrm{~J} / \mathrm{m}^{2}$ & & \\
\hline
\end{tabular}

Table 3 : Input data for 2D simulation of unstable crack extension

In order to have unstable crack propagation for $2 \mathrm{D}$ configurations presented in Figure 5 we use the numerical values proposed in Table 3. As explained in section 2.2.1, the standard QNA can't converge in this case due to the loss of convexity of the problem. An illustration of this convergence problem is given in Figure 13 where the equilibrium residual always increases when the crack extension starts and the convergence can't be achieved even after a large number of iterations. Results with the proposed algorithm for the smooth specimen tensile test are presented in Figure 14. As expected, the unstable crack extension leads to a sudden tensile stress drop with a total strain increment equal to zero (see Figure 14a). The convergence process of the fictive path loading algorithm during the unstable rupture (see Figure 14b) leads to 132 iterations. Virtual steps updates are done every $50^{\text {th }}$ iterations. After the first virtual step, the elastic energy decreases up to zero as damage progresses through the thickness. Crack extension starts at iteration 50 and crosses the thickness at iteration 100 as shown in Figure 14c with the damage field and the crack extension energy. In this case, the use of a uniform stress level at the crack initiation leads to a thickness of the damage zone without physical meaning as we don't expect a multilayer crack pattern in a brittle material. As presented in a further section, a non-uniform stress distribution at the crack initiation loading wouldn't lead to the same result. Despite the lack of physical meaning of 
the damage zone thickness, we can see that the fracture energy is still much smaller than the elastic energy at crack initiation, and other parameters (rupture stress, stress-strain curve, crack orientation, number of damage zones,...) are in good agreement with the expected results. In Table 4 a sensitivity analysis shows that the maximal load $\sigma_{\operatorname{Max}}$ is not mesh size dependent. In all these cases, the crack extension is unstable as expected if we consider a single crack and available elastic energy at crack initiation.

\begin{tabular}{|l|l|l|l|l|l|}
\hline $\mathrm{L}$ & mesh size $h$ & $\sigma_{\operatorname{Max}}(\mathrm{MPa})$ & Fracture energy $(\mathrm{J})$ & Elastic energy $(\mathrm{J})$ & Type of FE \\
\hline $1.5 \mathrm{xt}$ & $\mathrm{t} / 70$ & 163.5 & 0.156 & 0.627 & QUA4 \\
\hline $1.5 \mathrm{xt}$ & $\mathrm{t} / 50$ & 163.7 & 0.136 & 0.628 & QUA4 \\
\hline $1.5 \mathrm{xt}$ & $\mathrm{t} / 50$ & 164 & 0.13 & 0.630 & TRI3 \\
\hline $\mathrm{t}$ & $\mathrm{t} / 50$ & 163.7 & 0.119 & 0.419 & QUA4 \\
\hline $2 \mathrm{xt}$ & $\mathrm{t} / 50$ & 163.7 & 0.163 & 0.837 & QUA4 \\
\hline
\end{tabular}

Table 4 : Unstable crack extension simulation results for a tensile smooth specimen For the specimen "Cracked 1" of Table 2 the simulation results are given in Figure 15 and Table 5. In the latter a sensitivity analysis has been achieved. The crack extension starts at a maximum stress of 21.5 $\mathrm{MPa}$ and leads to an unstable rupture. As mentioned previously, a non-uniform stress distribution, due to the initial crack, leads to a damage zone thickness equal to one element. As shown in Table 5, the maximum load level is not mesh size dependent, this result as well as the computation of the $G$ integral confirm once again that the smeared crack model is equivalent to a Griffith criterion (see also size effect in Table 5). We can also confirm that the specimen size effect is in good agreement with the Griffith criterion when we compare different results for the same $\frac{a}{t}$ ratio.

\begin{tabular}{|l|l|l|l|l|l|l|}
\hline $\mathrm{t}(\mathrm{m})$ & $\mathrm{L}$ & mesh size & $\mathrm{a} / \mathrm{t}$ & Maximal load $(\mathrm{MPa})$ & $\mathrm{G}$ integral $\left(\mathrm{J} / \mathrm{m}^{2}\right)$ & Type of FE \\
\hline $0.125 \mathrm{e}-2$ & $1.5 \mathrm{xt}$ & $\mathrm{t} / 50$ & 0.5 & 21.5 & 9.2 & QUA4 \\
\hline $0.125 \mathrm{e}-2$ & $1.5 \mathrm{xt}$ & $\mathrm{t} / 70$ & 0.5 & 21.5 & 8.9 & QUA4 \\
\hline $0.125 \mathrm{e}-2$ & $1.5 \mathrm{xt}$ & $\mathrm{t} / 100$ & 0.5 & 22 & 9 & QUA4 \\
\hline $0.125 \mathrm{e}-2$ & $1.5 \mathrm{xt}$ & $\mathrm{t} / 50$ & 0.5 & 19 & 7.5 & TRI3 \\
\hline $0.125 \mathrm{e}-2$ & $1.5 \mathrm{xt}$ & $\mathrm{t} / 50$ & 0.25 & 36.8 & 9.4 & QUA4 \\
\hline $0.0625 \mathrm{e}-2$ & $1.5 \times \mathrm{xt}$ & $\mathrm{t} / 50$ & 0.5 & 28.5 & 7.4 & QUA4 \\
\hline
\end{tabular}

Table 5 : Unstable crack extension simulation results for a tensile cracked specimen

The FPLA step and its verification, as proposed in this new methodology (see section 2.4), are illustrated in Figure 16 for unstable rupture of the cracked specimen. In Figure 16a the convergence process is 
similar to the one obtained for the smooth specimen with a decrease of the equilibrium residual error occurring only when the crack extension is maximal and when the elastic energy is almost relaxed. In Figure 16b we can see that the convergence of the QNA, initialized with the FPLA solution, is reached after only 2 iterations. This verification confirms that the solution satisfies both the equilibrium condition and the material law. In Figure 16c, the QNA without initialization shows that no convergence seems possible even after a large number of iterations. In this last case the crack extension can't start, probably because the QNA is trapped in a local minimum of the equilibrium residual. According to the damage extension of Figure 16d and to the crack extension energy of Figure 16a, we can conclude that the FPLA solution is consistent with a progressive damage extension from the crack tip.

\subsection{Mixed mode loading}

A cracked specimen under mixed mode loading I and II has been simulated according the geometrical configuration presented in Figure 17a and input data given in Table 6. As shown in Figure 17b the crack initiation starts for a maximum shear stress of $23 \mathrm{MPa}$ in a direction oriented at an angle of $60^{\circ}$ compare to the initial crack plane. There is a stable crack extension up to $20 \%$ of the normalized displacement loading, and then an unstable crack extension reaches the bottom of the specimen and leads to a complete relaxation of the shear stress loading. After that, the tensile stress can increase up to a maximum value of $17 \mathrm{MPa}$ leading to an unstable crack extension at the bottom of the specimen, under a mode I loading, from the first mixed mode crack tip. A sensitivity analysis with respect to the ratio shear over tensile displacement loading has been achieved. This study shows that through this ratio one can have mode II, mode I or mixed mode I and II rupture types. The stress-displacement curve as well as the crack path are not mesh size dependent. However the finite element type and the spatial mesh distribution have a significant impact on crack path due to discrete approximation of the stress field as it is illustrated in Figure 18. In the latter one can see that in the mode II loading case the crack path orientation (red curve in Figure 18) depends on the finite element type. This problem seems to be related to an artificial texture effect brings by the maximal principal stress criterion and the finite element approximation of the stress field.

\begin{tabular}{|l|l|l|l|l|l|l|l|}
\hline$t(\mathrm{~m})$ & $L$ & $a$ & $E$ & $v$ & $G_{C}$ & $\sigma_{R}$ & $u_{x}$ \\
\hline $0,25.10^{-2}$ & $0,5 . \mathrm{t}$ & $0,5 . \mathrm{t}$ & $200 \mathrm{GPa}$ & 0.3 & $4 \mathrm{~J} / \mathrm{m}^{2}$ & $164,7 \mathrm{MPa}$ & $\frac{4,5 . \sigma_{R} \cdot L}{E}$ \\
\hline
\end{tabular}

Table 6 : Input data for 2D simulation under mixed mode loading

\subsection{Bi-material tensile test}

A bi-material tensile test has been simulated according to the geometrical configuration presented in Figure 19 and input data given in Table 7. The first material is considered to be elastic. The rupture can 
occur only in the second material with a toughness set at 2 or $1 \mathrm{~J} / \mathrm{m}^{2}$. For the crack initiation, we define a weak finite element located at the bottom of the specimen in material 2. In each case, we can see in Figure 19 that the crack initiation occurs at a tensile stress of $164 \mathrm{MPa}$ which is consistent with the rupture stress. For the smaller toughness case, a first unstable rupture occurs at the bottom of the specimen with a crack extension through one third of the bi-material interface. Then the crack extension goes on at the bi-material interface under a stable stage up to the end of the loading. For the $2 \mathrm{~J} / \mathrm{m}^{2}$ toughness case, the first unstable rupture stops at the bi-material interface. Then the crack extension continues along the interface, and new cracks, parallel to the first one, initiate regularly along the specimen length. These results are consistent with the ones proposed in reference [32] where the periodic length between two horizontal cracks has an inverse proportionality with the material toughness.

\begin{tabular}{|l|l|l|l|l|l|}
\hline$t=t_{1}=t_{2}(\mathrm{~m})$ & $L$ & $E_{1}=E_{2}$ & $v_{1}=v_{2}$ & $\sigma_{R_{2}}$ & $u_{y}$ \\
\hline $0,025.10^{-2}$ & $\mathrm{t}$ & $200 \mathrm{GPa}$ & 0.3 & $164,7 \mathrm{MPa}$ & $\frac{2 . \sigma_{R_{2}} \cdot L}{E}$ \\
\hline
\end{tabular}

Table 7 : Input data for 2D simulation of a bi-material tensile test

\subsection{D nuclear fuel simulation}

\subsubsection{Macroscopic fragmentation under thermal gradient}

In order to study nuclear fuel pellet fragmentation under irradiation we have developed a 3D finite element simulation of one single pellet fragment [1]. In this study, we propose an improvement of the simulation with a full pellet finite element model as presented in Figure 20. Isostatic boundary conditions are used in order to avoid rigid body motion of the fuel pellet. The loading is induced by a radial thermal radial gradient with a parabolic shape according higher temperatures at the center of the pellet. This thermal gradient is increased gradually in order to reach a maximal mean value of $-150{ }^{\circ} \mathrm{C} / \mathrm{mm}$ at the end of the loading. Other input data are given in Table 8 and simulation results are presented in Figure 21. The crack extension is unstable and starts at a thermal gradient of $-25{ }^{\circ} \mathrm{C} / \mathrm{mm}$ with initiation sites localized on the outer part of the pellet where the tensile thermal stresses are maximal. At this stage, there are three pellet fragments with crack planes oriented perpendicularly to the circumferential direction. It is worth noting that although the tensile circumferential stress is almost constant on the outer part of the pellet, we don't need to introduce a weak element to have damage localization leading to a first crack. In this case, the stress scattering due to numerical noise is enough to start the localization of the damage initiation before crack extension. A higher thermal gradient loading tends to increase the extension of the existing cracks and the connections between cracks including the ones coming from new initiation sites. In Figure 21e) the pellet fragmentation has been illustrated with an amplification of the displacement field and a suppression of the damaged elements. In this figure, we can see that the thermal gradient loading on 
the fragmented pellet leads to the well-known pellet hour-glass shape, as the phenomenon was established through a simplified 3D model and experimental results in the framework of pellet cladding interaction studies [1]. If we compare the crack pattern to experimental observations, we can conclude that the number of pellet fragments and their shape are representative of the beginning of irradiation.

For mesh sensitivity analysis purpose, three different mesh refinements have been used with 3340,7658 and 24397 nodes respectively. Results are presented in Figure 22 with the pellet fragmentation obtained for the maximal thermal gradient. The local softening modulus of the damage model has been computed according Equation (24) in order to have a material toughness equal to $17 \mathrm{~J} / \mathrm{m}^{2}$. The results are qualitatively consistent for the three meshes if we consider the fragment size and the hour glass shape of the pellet. The cracked surfaces, estimated through the volume of the damaged element, are 5.4, 4.9 and $5.1 \mathrm{~cm}^{2}$ for mesh 1 to 3 respectively. The variation between mesh 2 and 3 seems reasonable, however mesh convergence should be confirmed with higher refinements. Computation times are 1, 3 and 18 hours for mesh 1 to 3 respectively. The numbers of time steps used for meshes 1 to 3 are 2400, 3400 and 5500 respectively. For mesh 3 computation, there is 140 FPLA steps and a mean number of QNA iterations of 10. The FPLA steps for the first unstable cracks required a high number of iterations (between 300 and 400).

\begin{tabular}{|l|l|l|l|l|l|l|}
\hline Fuel pellet & \multicolumn{4}{|l|}{ Material properties } \\
\hline radius & height & $\begin{array}{l}\text { Young } \\
\text { modulus }\end{array}$ & $\begin{array}{l}\text { Poisson } \\
\text { ratio }\end{array}$ & $\begin{array}{l}\text { Rupture } \\
\text { stress }\end{array}$ & Toughness & $\begin{array}{l}\text { Thermal } \\
\text { expansion }\end{array}$ \\
\hline $4 \mathrm{~mm}$ & $7 \mathrm{~mm}$ & $\begin{array}{l}200000 \\
\mathrm{MPa}\end{array}$ & 0.3 & $\begin{array}{l}100 \\
\mathrm{MPa}\end{array}$ & $10 \mathrm{~J} / \mathrm{m}^{2}$ & $1 \mathrm{e}-05$ \\
\hline
\end{tabular}

Table 8 : Input data for fuel pellet fragmentation simulation

\subsubsection{Microscopic fragmentation under fission gas pressure loading.}

Under irradiation, gaseous fission products tend to precipitate in small bubbles and induced internal stresses in the porous material. To study the risk of fuel pellet microscopic fragmentation, we have developed a 3D volume element model of the nuclear fuel [2]. The finite element mesh (see Figure 23), derived from a MOX fuel microstructure [33], is based on a periodic volume element of the porous material. Bubble size statistical distribution is defined with post irradiation micrographic examinations, and bubble spatial localization is derived from a Random Sequential Addition algorithm. Input parameter are given in Table 9. As the loading due to pressure driven forces on the crack surfaces is not represented in the finite element simulation, we have to use a reduced value of the real material toughness. This effective toughness has been estimated thanks to the difference between the material toughness and the work due the pressure on the surface crack. This leads to a very small value of $0.001 \mathrm{~J} / \mathrm{m}^{2}$ as given in 
Table 9. In order to avoid an indeterminacy of the static solution under Neumann boundary condition, the pressure loading is replaced by a strain control loading. The latter is defined through a fictive thermal expansion of the bubble gas, itself considered as a linear elastic material with a shear modulus much smaller than the bulk modulus. Numerical results are presented in Figure 24 with an amplification of the displacement field and a suppression of damaged elements. As presented in Figure 24a, the mean bubble pressure, induced by the fictive thermal loading, increases up to $120 \mathrm{MPa}$ when a first unstable crack extension occurred. After the first crack extension the pressure is no more homogeneous in bubbles. Bubbles crossed by or near a crack plane have a reduced pressure due to the softening of damaged material. This leads to a mean pressure drop for each new crack extension as shown in Figure 24a. Through these results, we can understand the fuel fragmentation mechanism which depends on the material microstructure and the pressure induced by gaseous fission products.

\begin{tabular}{|l|l|l|l|l|l|l|}
\hline \multicolumn{2}{|l|}{ Fuel Volume element } & Material properties \\
\hline Size & $\begin{array}{l}\text { Bubble volumic } \\
\text { fraction ratio }\end{array}$ & Young modulus & $\begin{array}{l}\text { Poisson } \\
\text { ratio }\end{array}$ & $\begin{array}{l}\text { Rupture } \\
\text { stress }\end{array}$ & $\begin{array}{l}\text { Effective } \\
\text { material } \\
\text { toughness }\end{array}$ & $\begin{array}{l}\text { Fictitious } \\
\text { thermal } \\
\text { expansion }\end{array}$ \\
\hline $9 \mu \mathrm{m}$ & $8 \%$ & $200000 \mathrm{MPa}$ & 0.3 & $\begin{array}{l}100 \\
\mathrm{MPa}\end{array}$ & $0.001 \mathrm{~J} / \mathrm{m}^{2}$ & 1. \\
\hline
\end{tabular}

Table 9 : Input data for fuel microscopic fragmentation

\subsubsection{Discussion}

These 3D applications demonstrate the robustness of the proposed algorithm to simulate a rupture process involving multiple crack initiation sites and complex crack extension patterns. Thanks to the continuous damage model this approach doesn't need a specific mesh refinement method. Computation times obtained with personal computers are reasonable (between 1 and 2 days) compared to the complexity of the rupture process. Such simulations can now be achieved to study with more details the fuel fragmentation process at macroscopic and microscopic scales, in order to have a better understanding of its impact on the fuel element structural integrity.

\section{CONCLUSIONS}

A new numerical approach has been proposed for unstable rupture simulation in brittle material. It is based on a three-steps algorithm: a time step refinement associated to a quasi-Newton algorithm (QNA) to detect the non-convergence only due to an unstable crack extension, a fictive path loading algorithm (FPLA) to reach the static post instability solution and finally a quasi-Newton algorithm initialized by the solution obtained from the fictive path loading algorithm to ensure the final solution to verify the quasi20 
static equilibrium as well as the behaviour law. This method doesn't need a fully dynamic description of the rupture and can overcome the discontinuous solution problems induced by the quasi-static equilibrium formulation.

An application of this new method has been presented for a smeared crack model, based on a local continuous damage formulation, devoted to brittle nuclear fuel rupture simulation. The robustness of the algorithm has been demonstrated through a set 2D and 3D simulation results.

First, we can conclude that the time step refinement process is efficient for the detection of unstable crack propagation steps. Thanks to the time step refinement the convergence of the QNA can be reached for softening behaviour under stable crack condition, and hence the FPLA is activated just at the beginning of the unstable crack growth. The FPLA, which can be easily implemented on an existing Newton type algorithm, provides a real improvement to simulate unstable crack extension in some cases where convergence is not possible with a QNA. The method has not been directly compared to other algorithms mentioned in the bibliography, nevertheless computation times seem reasonable regarding the complexity of applications proposed in the paper. The third step of the proposed methodology provides a simple verification to make sure that the FPLA has been used in an appropriate situation.

Further perspectives can be considered to improve the convergence of the algorithm such a sensitivity analysis to the numerical parameters used to control the time step refinement or the number of iterations between virtual steps. Comparison with other approaches can also be achieved through the extension to other damage models. A work is currently in progress to apply the proposed numerical strategy for a nonlocal damage model coupled to a phase field formulation. The first results are extremely encouraging. 


\section{REFERENCES}

[1] B. Michel, C. Nonon, J. Sercombe, F. Michel, V. Marelle, Simulation of the Pellet Cladding Interaction phenomenon with the PLEIADES fuel performance software environment, Nuclear Technology 182 (2), 124-137, 2013.

[2] C. Esnoul, R. Largenton, C. Petry, J.C. Michel, B. Michel, A. Bouloré, Use of micromechanical approach to investigate transient fuel fragmentation mechanisms, TopFuel 2015 Conference Proceedings - oral presentations - Part II, pp. 476-485, 2015.

[3] B. Michel, J. Sercombe, G. Thouvenin, R. Chatelet, 3D fuel cracking modelling in pellet cladding mechanical interaction, Engineering Fracture Mechanics 75 3581-3598, 2008

[4] T. Helfer, Introducing the open-source MFront code generator : application to mechanical behaviours and material knowledgemanagement within the PLEIADES fuel element modelling plateform, Computers and Mathematics with Applications, accepted the $24^{\text {th }}$ June 2015.

[5] http://www-cast3m.cea.fr.

[6] B. Michel, J. Sercombe, G. Thouvenin, A new phenomenological criterion for pellet-cladding interaction rupture, Nuclear Engineering and Design 238 (7), 1612-1628, 2008.

[7] J. Sercombe, M. Agard, C. Struzik, B. Michel, G. Thouvenin, C Poussard, 1D and 3D analyses of the Zy2 SCIP BWR ramp tests with the fuel codes METEOR and ALCYONE, Nuclear Engineering and Technology, 41(2009) pp. 187-198

[8] G.A. Francfort, J.J. Marigo, Revisiting Brittle Fracture as an Energy Minimization Problem, Journal of the Mechanics and Physics of Solids 46 (1998): 1319-42

[9] B. Bourdin, G.A. Francfort, and J.J. Marigo, Numerical Experiments in Revisited Brittle Fracture, Journal of the Mechanics and Physics of Solids 48 (2000): 797-826

[10] A. Hanen, J.J. Marigo, C. Maurini, Regularized Formulation of the Variational Brittle Fracture with Unilateral Contact: Numerical Experiments, Journal of the Mechanics and Physics of Solids 57 (2009): 1209-29

[11] B. Halphen, Q.S. Nguyen, Sur Les Matériaux Standard Généralisés, Journal de Mécanique 14 (1975): 39-63

[12] P. Germain, Q.S. Nguyen, P. Suquet, Continuum Thermodynamics, Journal of Applied Mechanics 50 (1983): 1010-20

[13] E. Lorentz, S. Andrieux, Analysis of Non-Local Models Through Energetic Formulations, International Journal of Solids and Structures 40 (2003): 2905-36

[14] X. Teng, Numerical Prediction of Slant Fracture with Continuum Damage Mechanics, Engineering Fracture Mechanics 75 (2008): 2020-41

[15] V. Acary, F. Dubois, Y. Monerie, Nonsmooth fracture dynamics using a cohesive zone approach, HAL Id: inria-00110560 
[16] J.P. Bobineau, B. Maurel, L. Morançay, AMS and Multi-Domain Method in RADIOS, Giens (2013), France

[17] T. Belytschko, Nonlinear Finite Elements for Continua and Structures, Chichester New York: Wiley-Blackwell (2000)

[18] J.A. Oliver, E Huespe, JC Cante, An Implicit/explicit Integration Scheme to Increase Computability of Non-Linear Material and Contact/friction Problems, Computer Methods in Applied Mechanics and Engineering 197 (2008) 1865-89

[19] C. Miehe, M. Hofacker, F. Welschinger, A Phase Field Model for Rate-Independent Crack Propagation: Robust Algorithmic Implementation Based on Operator Splits, Computer Methods in Applied Mechanics and Engineering 199 (2010): 2765-78

[20] T.T. Nguyen, J. Yvonnet, Q.Z. Zhu, M. Bornert, C. Chateau, A Phase Field Method to Simulate Crack Nucleation and Propagation in Strongly Heterogeneous Materials from Direct Imaging of Their Microstructure, Engineering Fracture Mechanics 139 (2015): 18-39

[21] T.T. Nguyen, J. Yvonnet, Q.Z. Zhu, M. Bornert, C. Chateau, A Phase-Field Method for Computational Modeling of Interfacial Damage Interacting with Crack Propagation in Realistic Microstructures Obtained by Microtomography, Computer Methods in Applied Mechanics and Engineering (2016).

[22] E. Lorentz, A mixed interface finite element for cohesive zone models, Comput. Methods Appl. Mech. Engrg. 198 (2008) 302-317

[23] N. Moes, T. Belytschko, Extended finite element method for cohesive crack growth, Engineering Fracture Mechanics 69 (2002) 813-833.

[24] J. Lemaitre, A Continuous Damage Mechanics Model for Ductile Fracture, Journal of Engineering Materials and Technology, 107(1985)83-89 .

[25] J. Mazars, A description of micro and macroscale damage of concrete structures, Engineering Fracture Mechanics, 25 (1986) 729-737.

[26] G. Pijaudier-Cabot, Z.P. Bazant, Nonlocal Damage Theory, Journal of Engineering. Mechanics, ASCE, 1987, Vol. 113(10), pp. 1512-1533.

[27] E. Lorentz, P. Badel, A new path-following constraint for strain-softening finite elements simulations, Int. J. Numer. Methods Engrg., 60 (2004), pp. 499-526

[28] J. Soulacroix, Micromechanical approach of behavior of Uranium dioxide nuclear fuel, Ph-D report, 2014.

[29] H.G. deLorenzi, On the energy release rate calculations by the finite element method, Eng. Fract. Mech., 21(1985)pp. 129-143.

[30] I. Ramière, Th. Helfer, Iterative residual-based vector methods to accelerate fixed point iterations, Computers\&Mathematics with Applications, Volume 70, Issue 9, November 2015, Pages 2210 2226. 
[31] D.G. Anderson, Iterative Procedures for Nonlinear Integral Equations, Journal of the Association for Computing Machinery 12 (4) (1965) 547-560

[32] G.A. Francfort, J.-J. Marigo, Vers une théorie énergétique de la rupture fragile, C. R. Mecanique 330 (2002) 225-233.

[33] R. Largenton, J.C. Michel, P. Suquet, Extension of the Nonuniform Transformation Field Analysis to linear viscoelastic composites in the presence of aging and swelling, Mechanics of Materials 73 (2014) 76-100. 
6. FIGURES

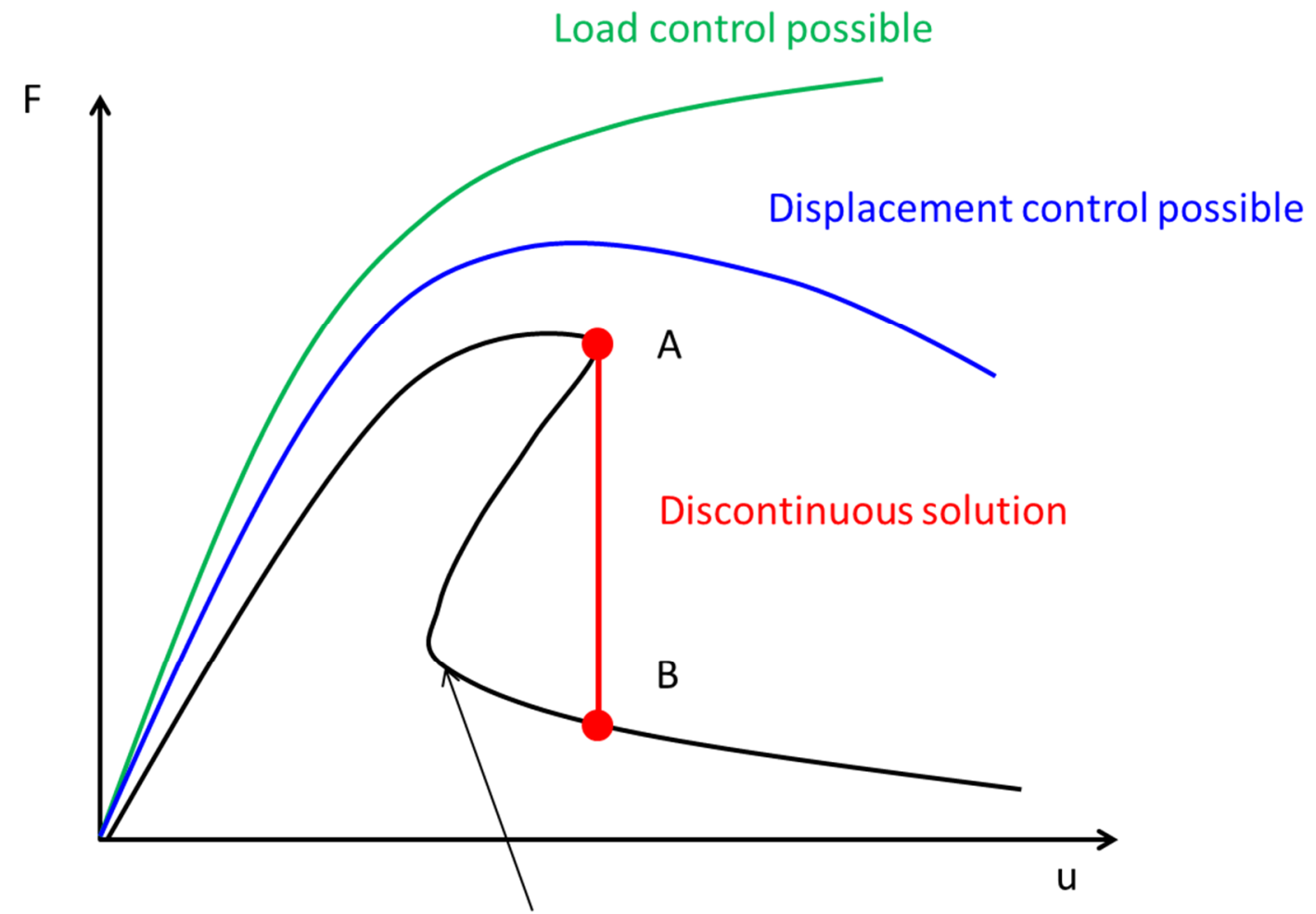

snap-back needed for the quasi static equilibrium

Figure 1 : Quasi static snap back under unstable crack extension and path following constraint solution. 


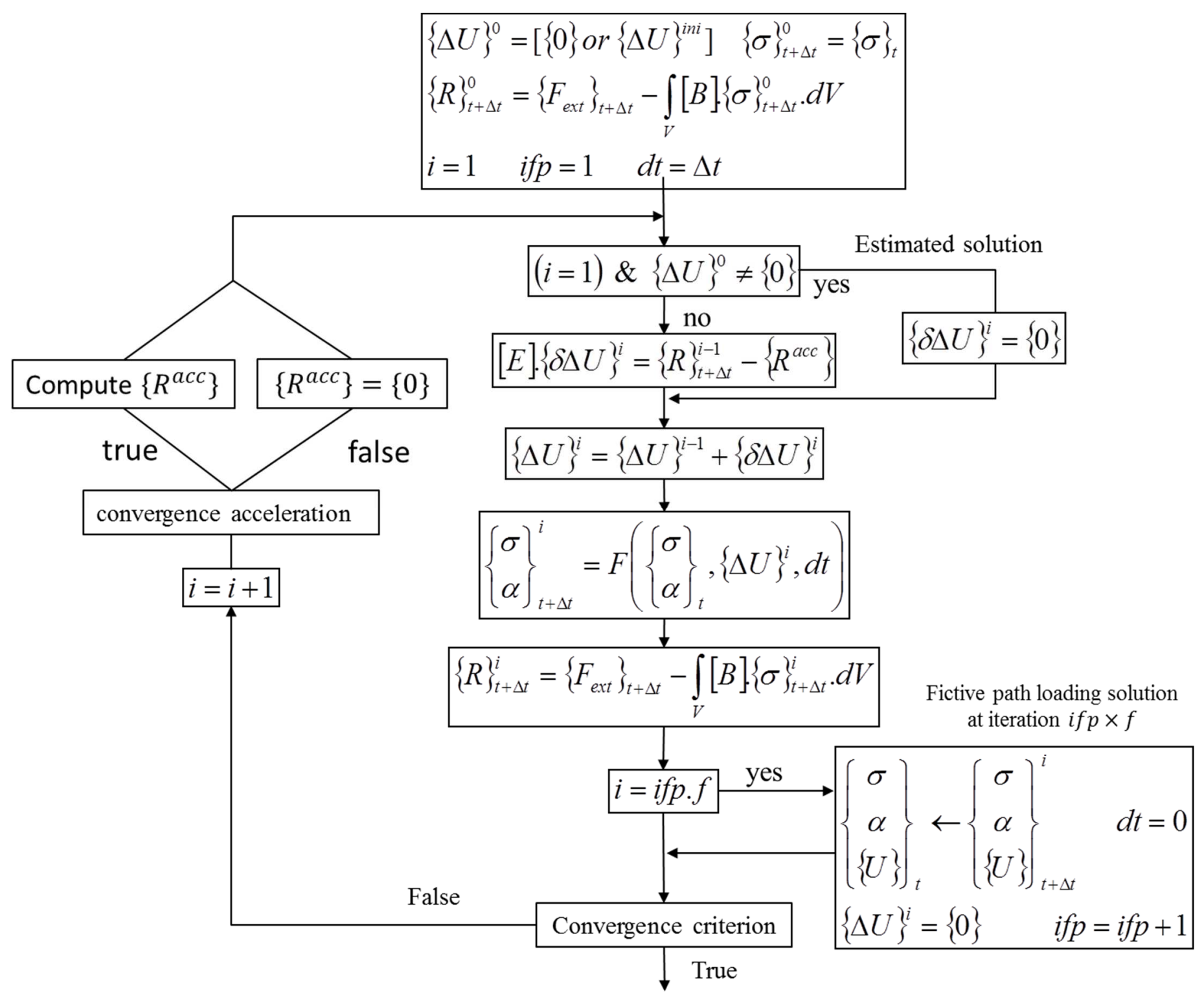

Figure 2 : Quasi Newton Algorithm of the Cast3M finite element software. 


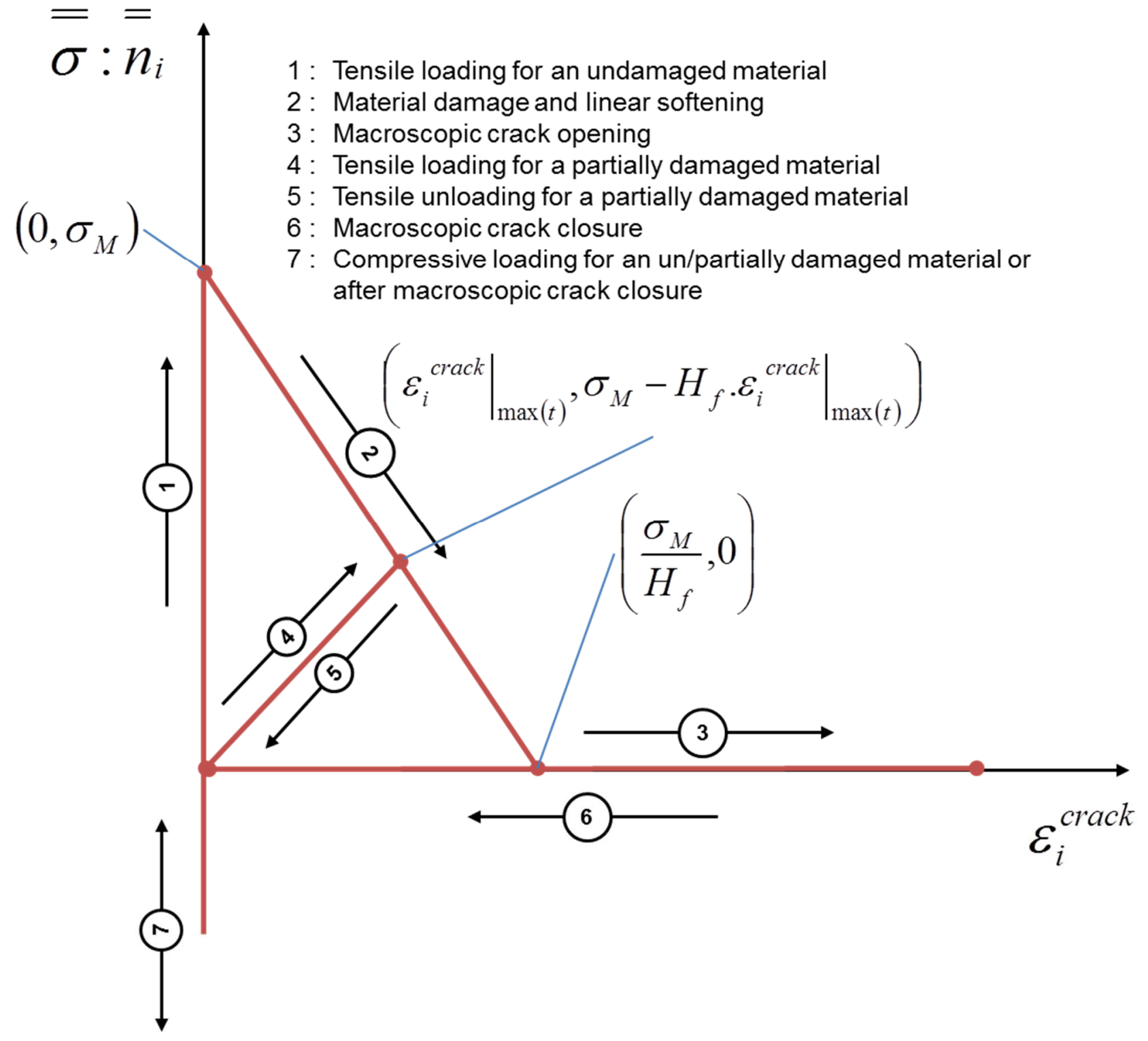

Figure 3 : Smeared crack model damage law

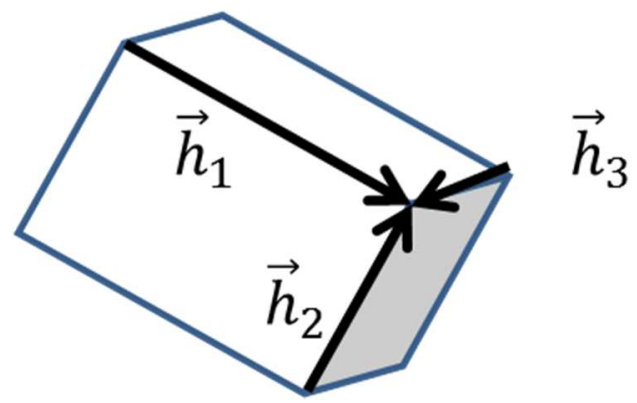

$$
\vec{h}=\vec{h}_{1}+\vec{h}_{2}+\vec{h}_{3}
$$

Figure 4 : Mesh size vector 


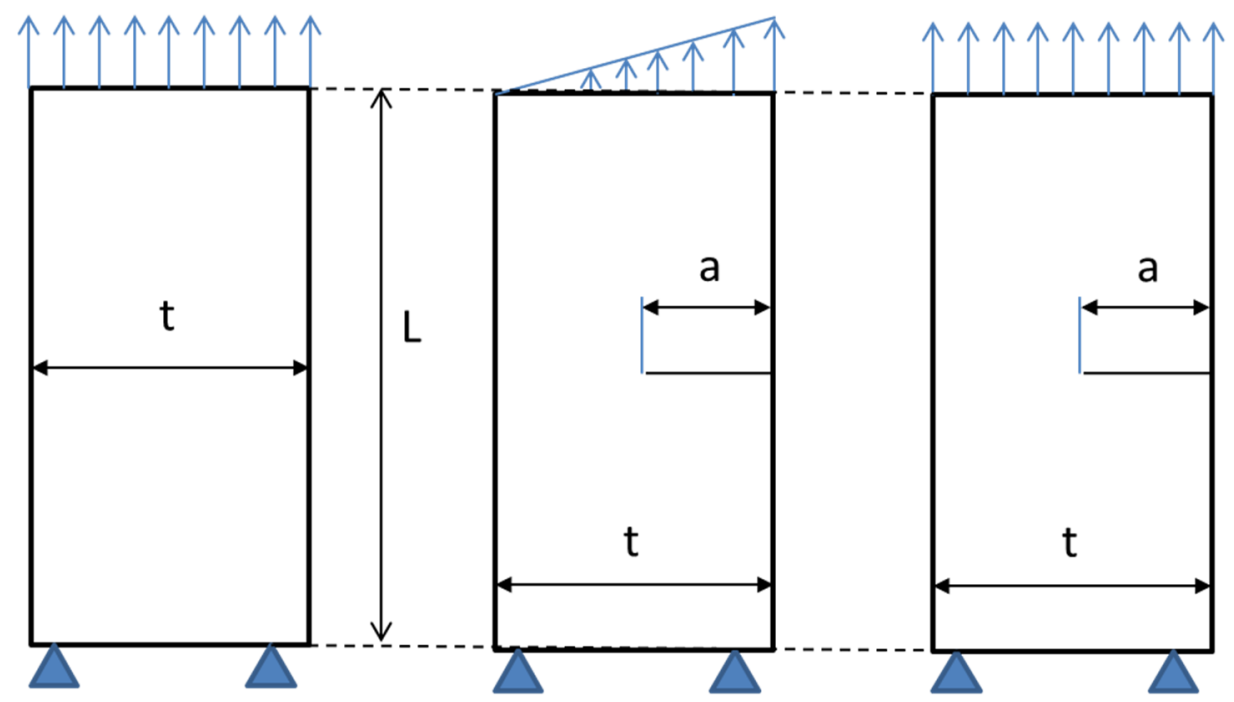

Smooth specimen Cracked specimen $1 \quad$ Cracked specimen 2

Figure $5: 2 \mathrm{D}$ configurations used to test the smeared crack model assessment. 


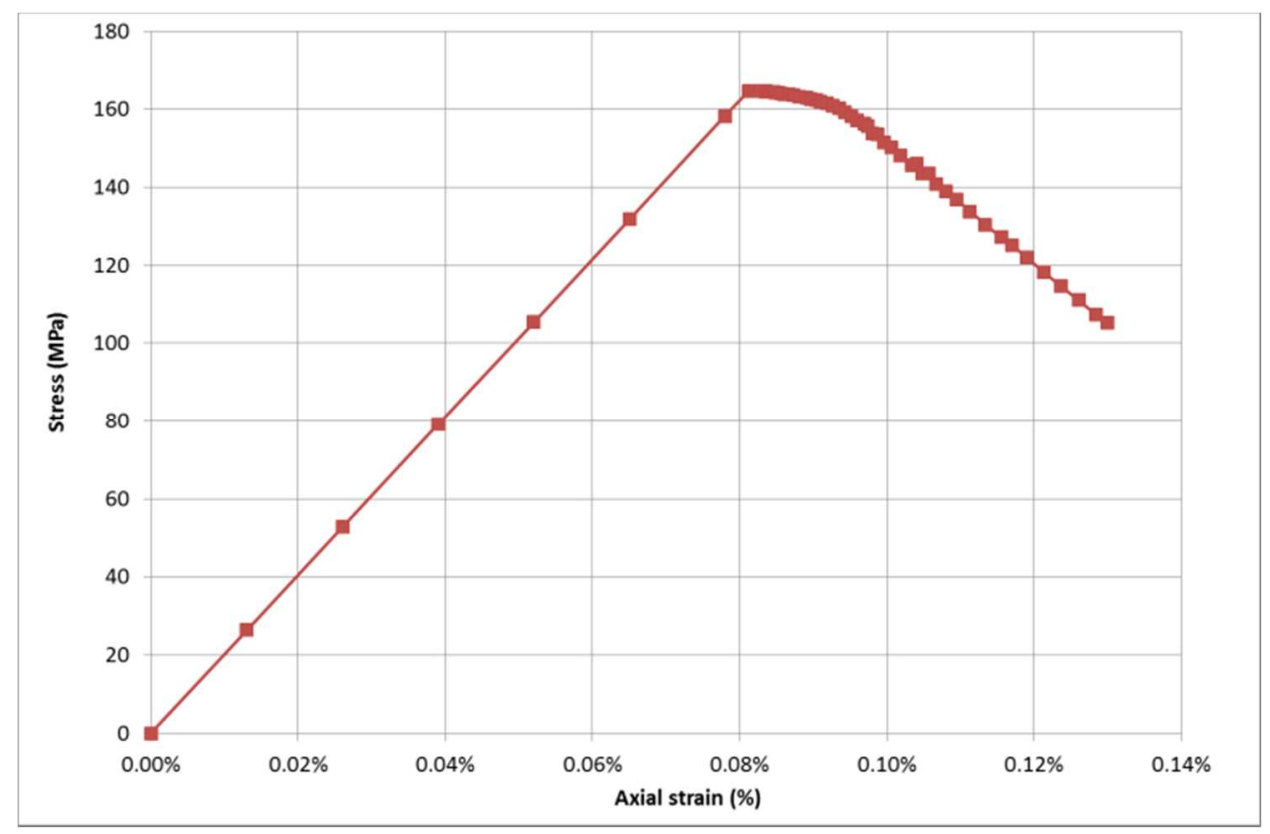

a) stress-strain curve

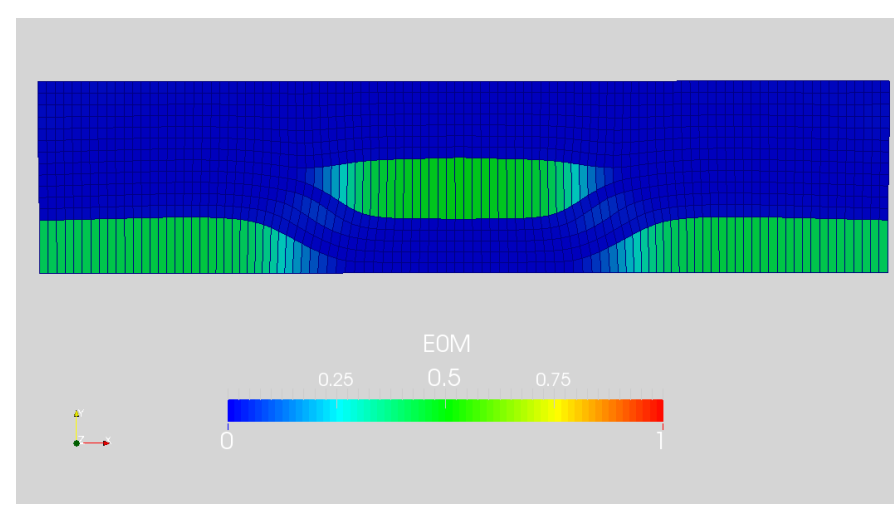

b) Damage extension (strain loading $0.13 \%$ )

$($ mesh size $=\mathrm{t} / 100)$

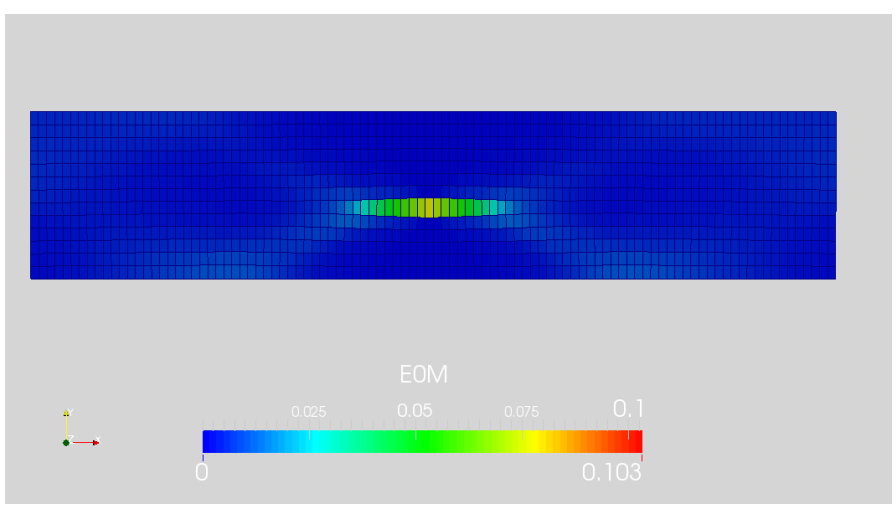

d) Damage extension (strain loading $0.06 \%$ )

$($ mesh size $=\mathrm{t} / 100)$

Figure 6 : Stable crack propagation results for the smooth specimen and uniform displacement boundary conditions (displacement amplification factor=100).

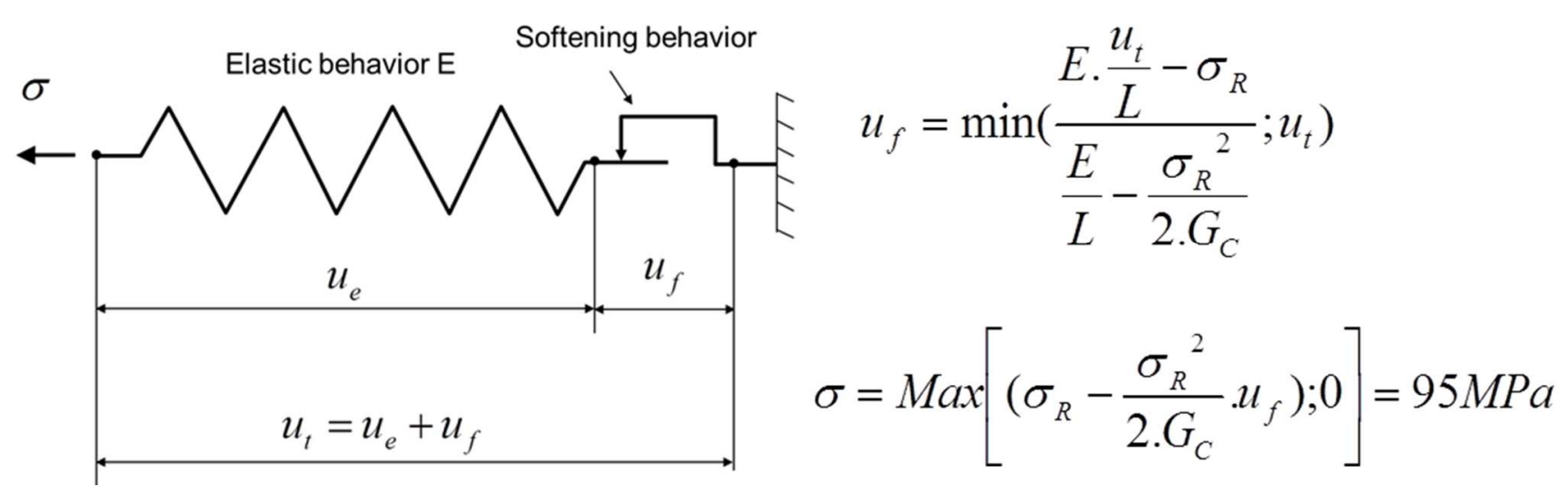


Figure 7 : Cohesive zone model and analytical solution for the smooth specimen rupture.

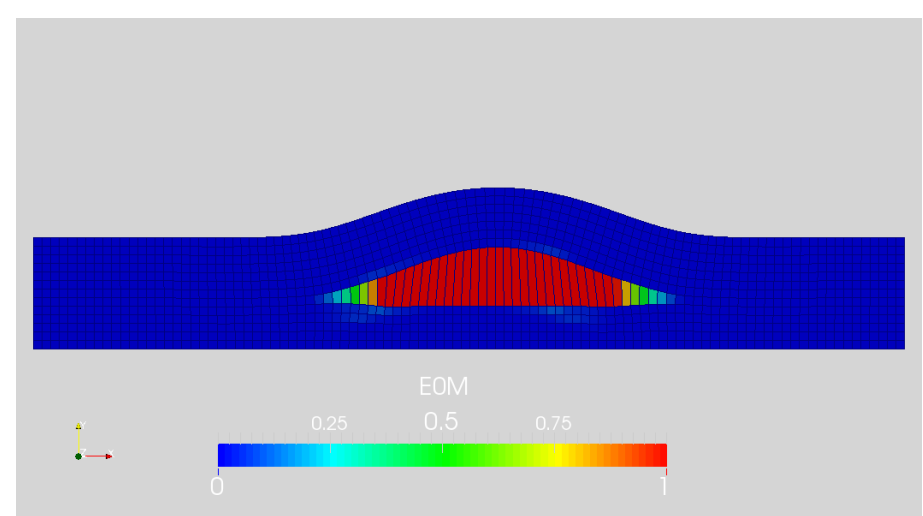

a) Damage extension (strain loading $0.13 \%$ ) $($ mesh size $=\mathrm{t} / 100)$

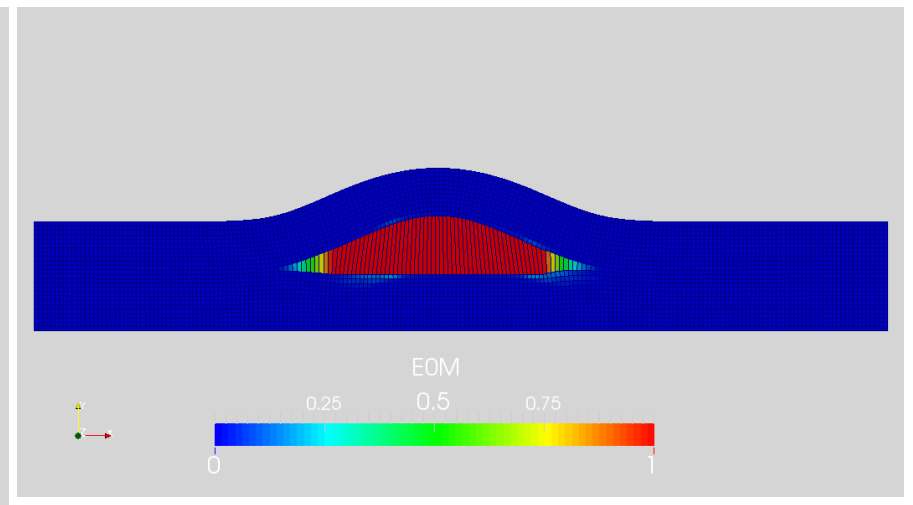

b) damage extension (strain loading $0.13 \%$ ) $($ mesh size $=\mathrm{t} / 200)$

Figure 8 : Damage extension for the smooth specimen with homogeneous stress boundary conditions (displacement amplification factor=100).

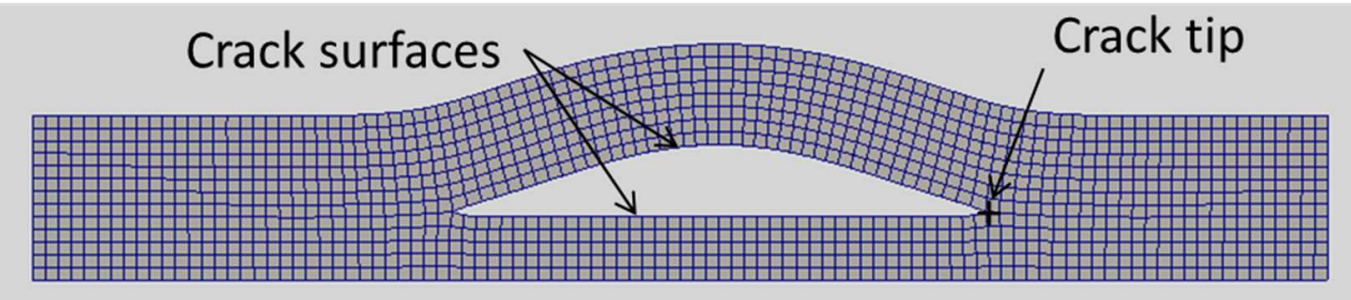

\begin{tabular}{|l|l|l|}
\hline mesh & crack size $(\mathrm{a} / \mathrm{t})$ & $\mathrm{G}\left(\mathrm{J} / \mathrm{m}^{2}\right)$ \\
\hline $\mathrm{t} / 100$ & 0.42 & 41.5 \\
\hline $\mathrm{t} / 200$ & 0.35 & 51.3 \\
\hline
\end{tabular}

Figure 9 : Energy restitution rate computation for the smooth specimen with homogeneous stress boundary conditions (displacement amplification factor=100). 


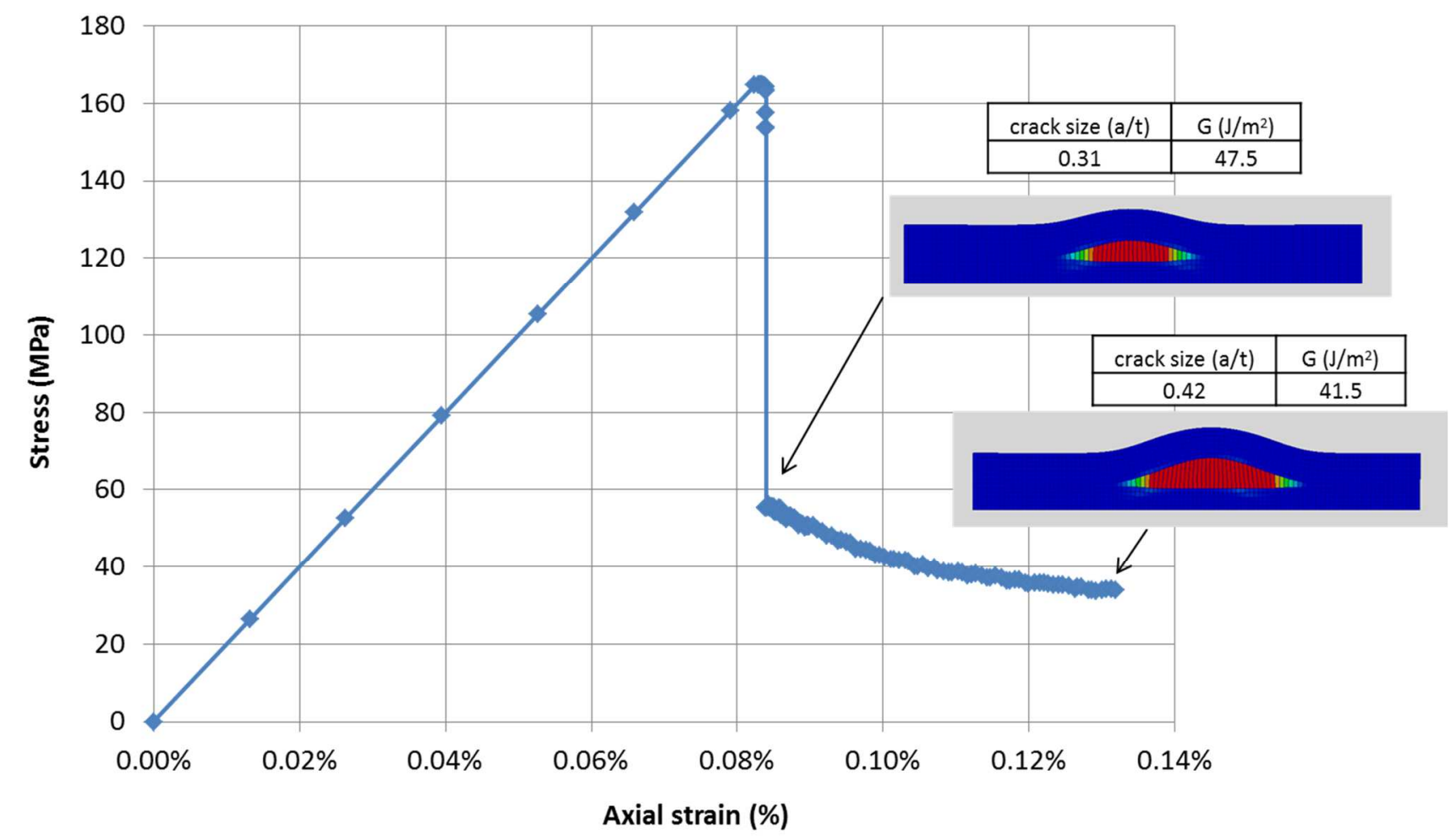

Figure 10 : Strain stress curve for the smooth specimen with homogeneous stress boundary conditions (displacement amplification factor=100) (mesh size $\mathrm{t} / 100)$. 


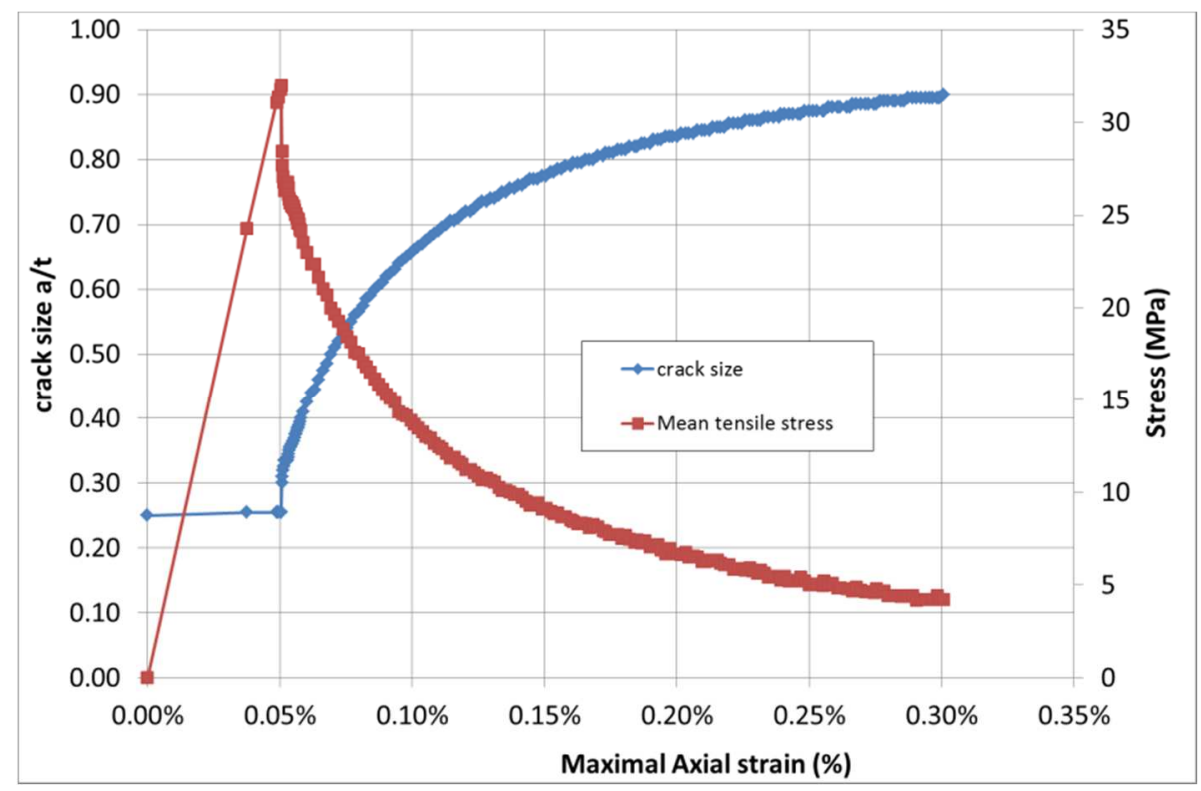

a) Crack extension and stress-strain curve

b) Normalized crack strain opening (displacement amplification factor=100)

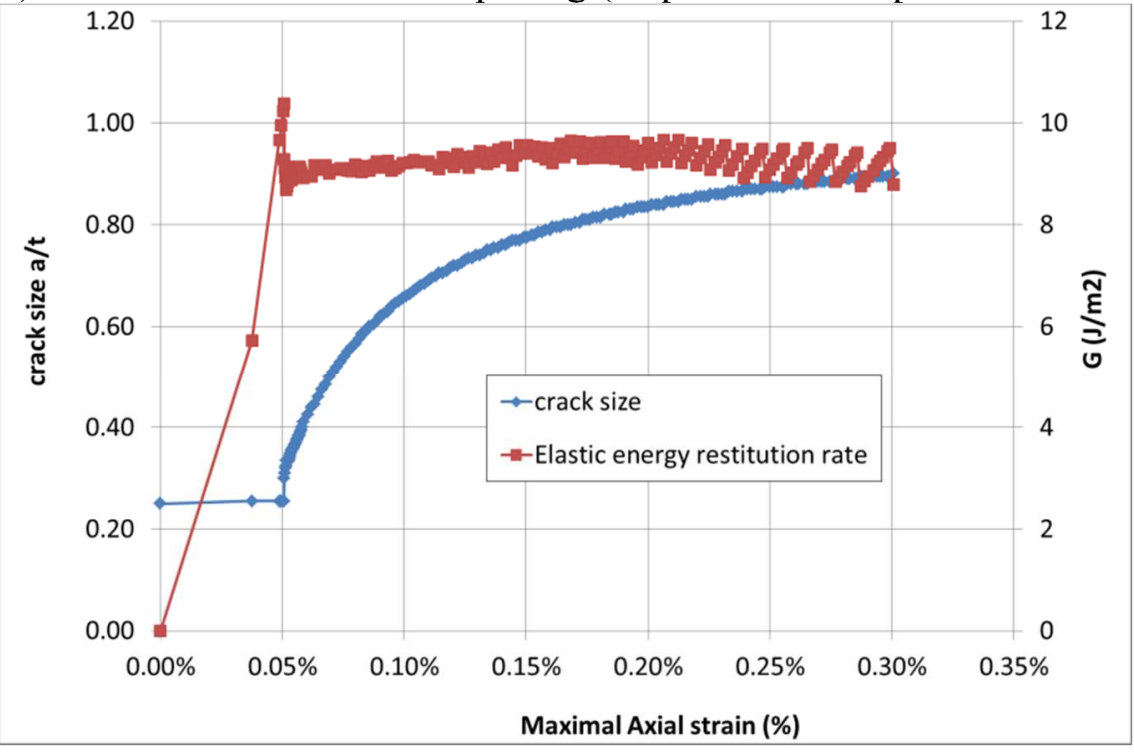

c) Crack extension and elastic energy restitution rate for the initial crack

Figure 11 : Stable crack propagation results for the cracked specimen $($ mesh size $=t / 200)$. 


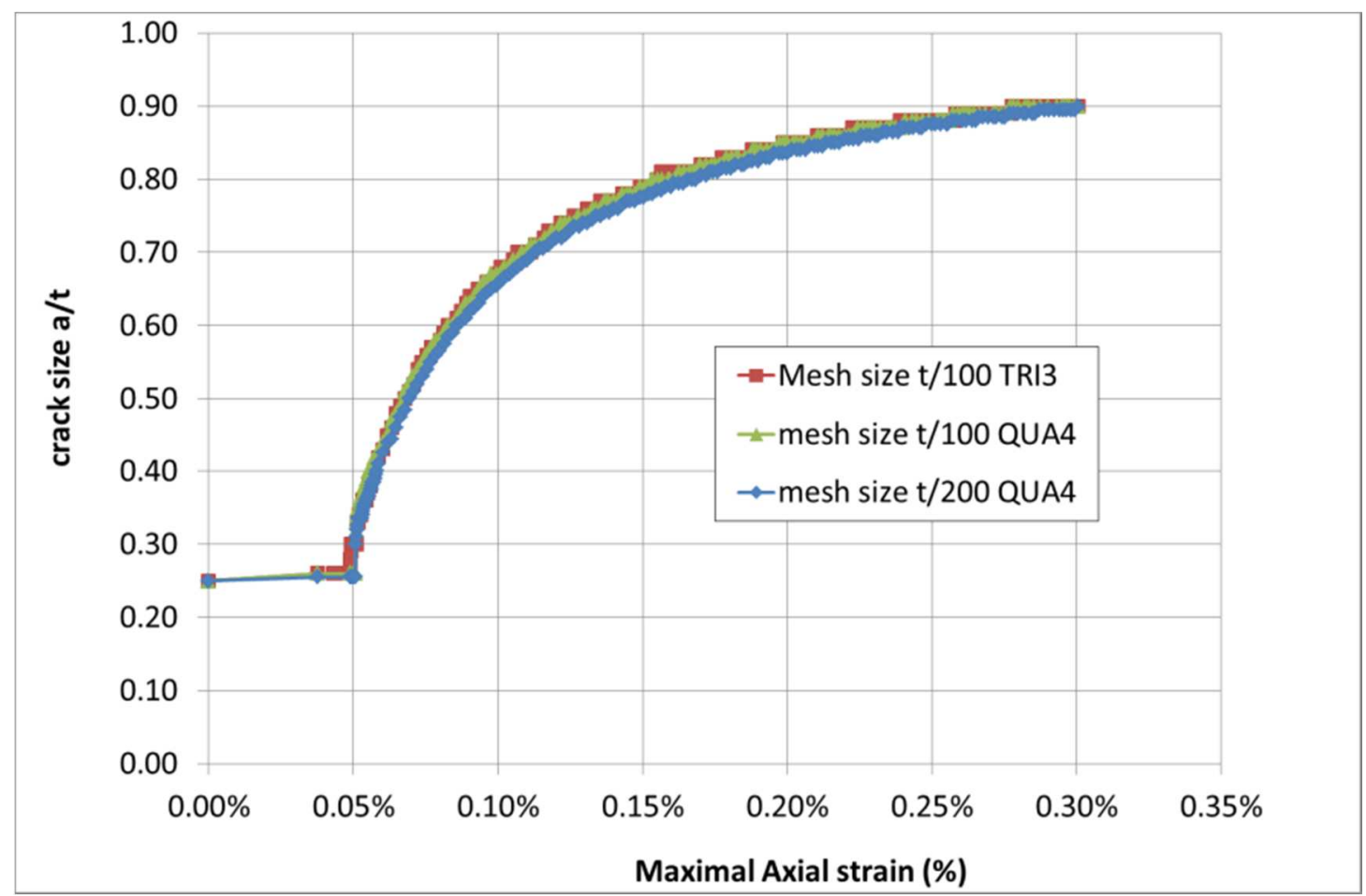

Figure 12 : Mesh size sensitivity analysis for stable crack propagation in the cracked specimen.

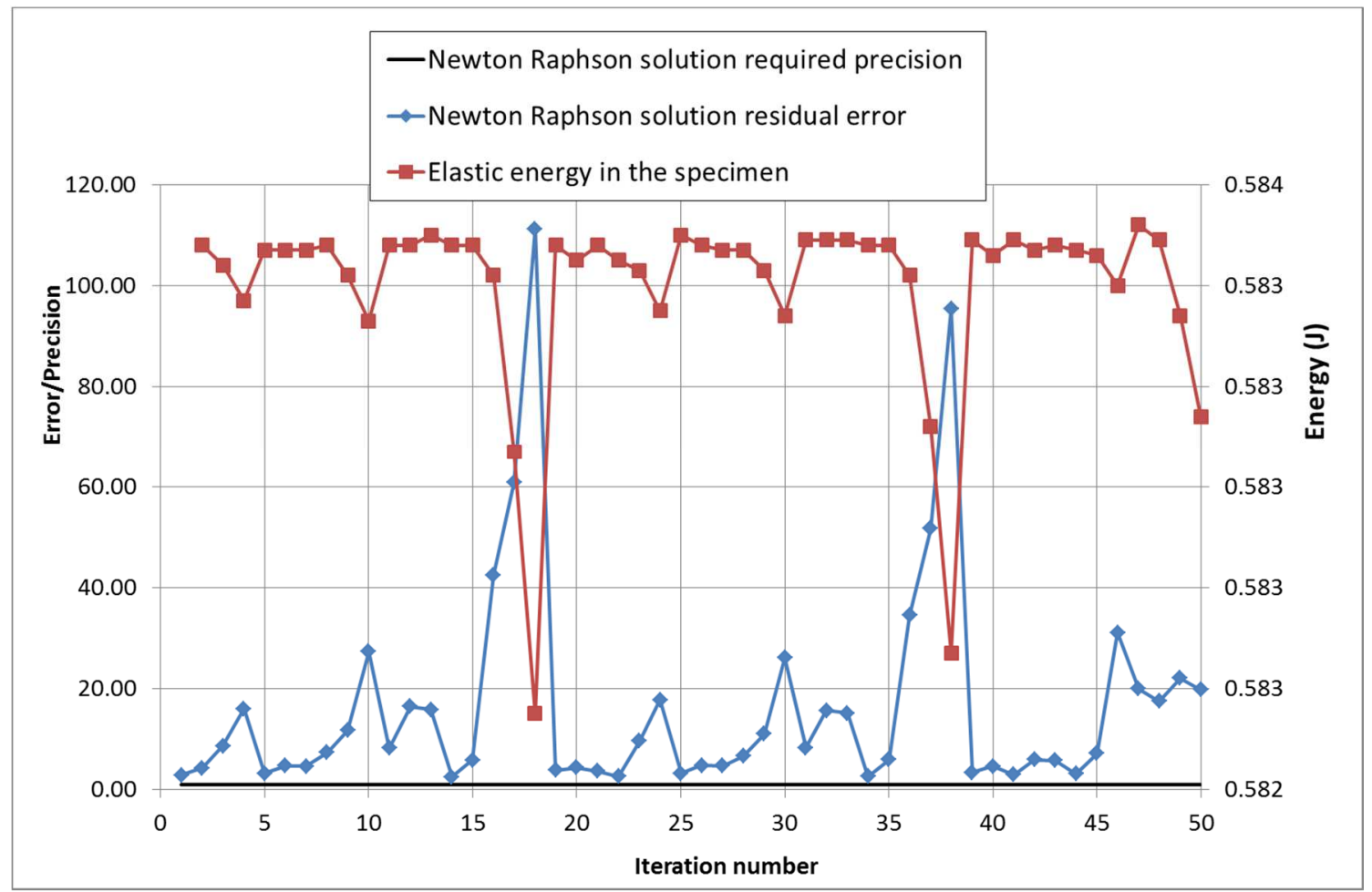

Figure 13 : Residual nodal forces of the Cast3M Quasi-Newton algorithm in the case of unstable crack extension. 


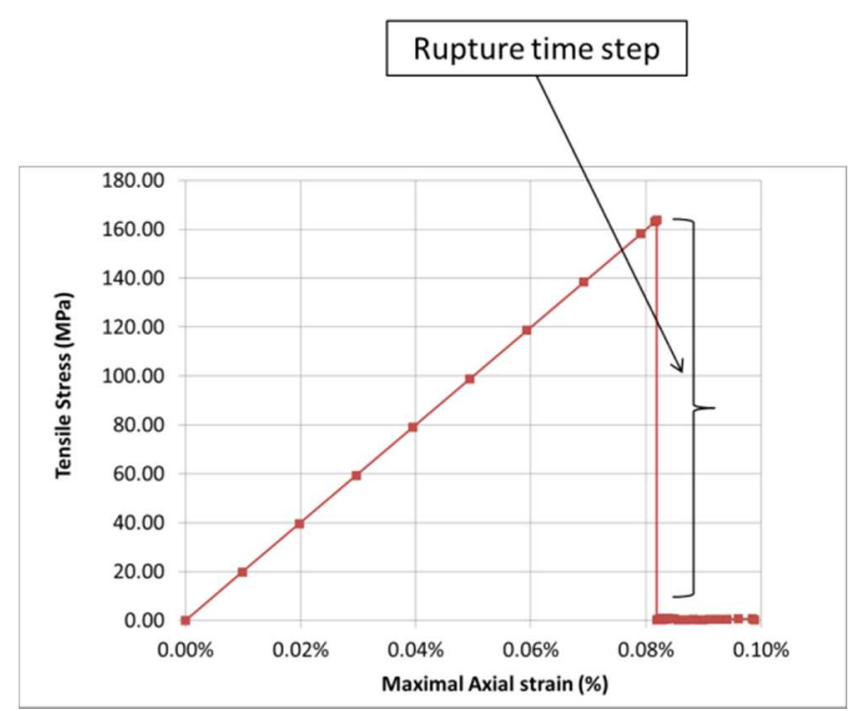

a) Stess-strain curve

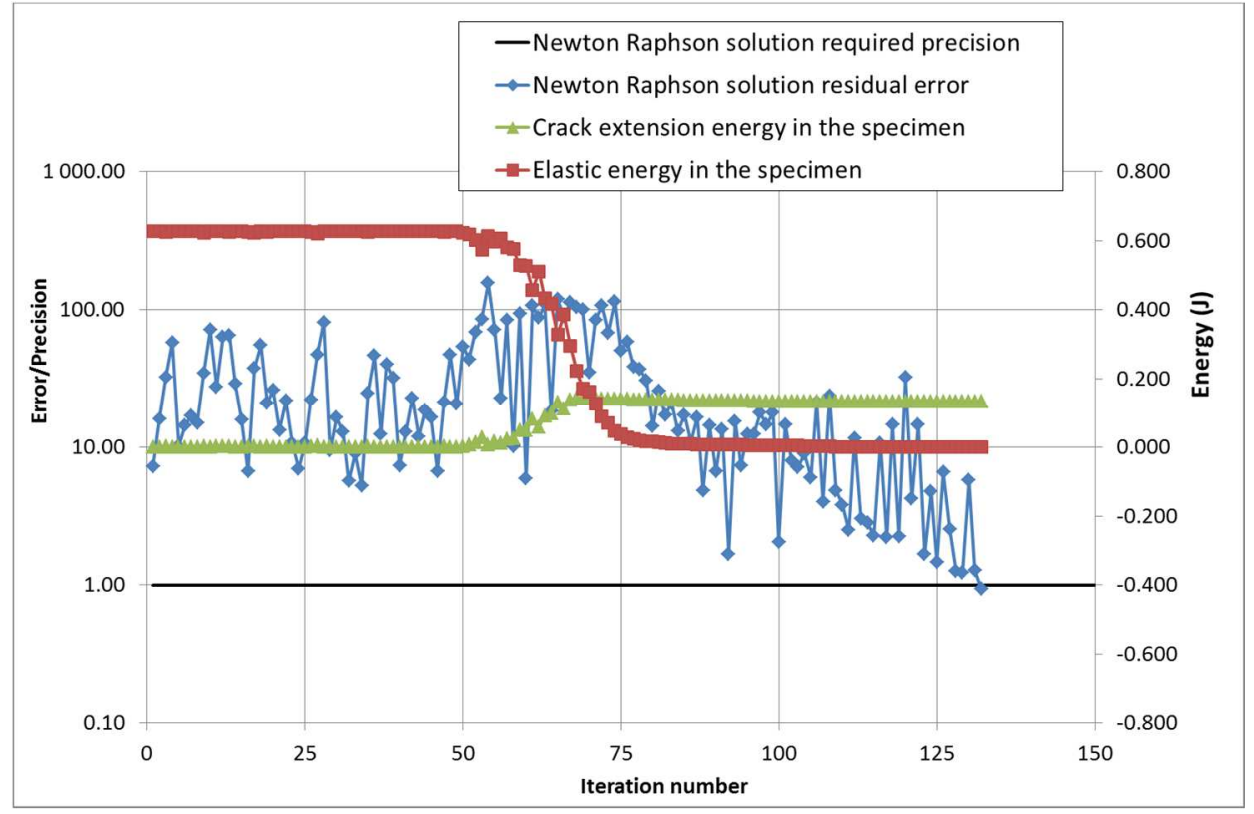

b) Convergence of the fictive path loading algorithm during the rupture time step

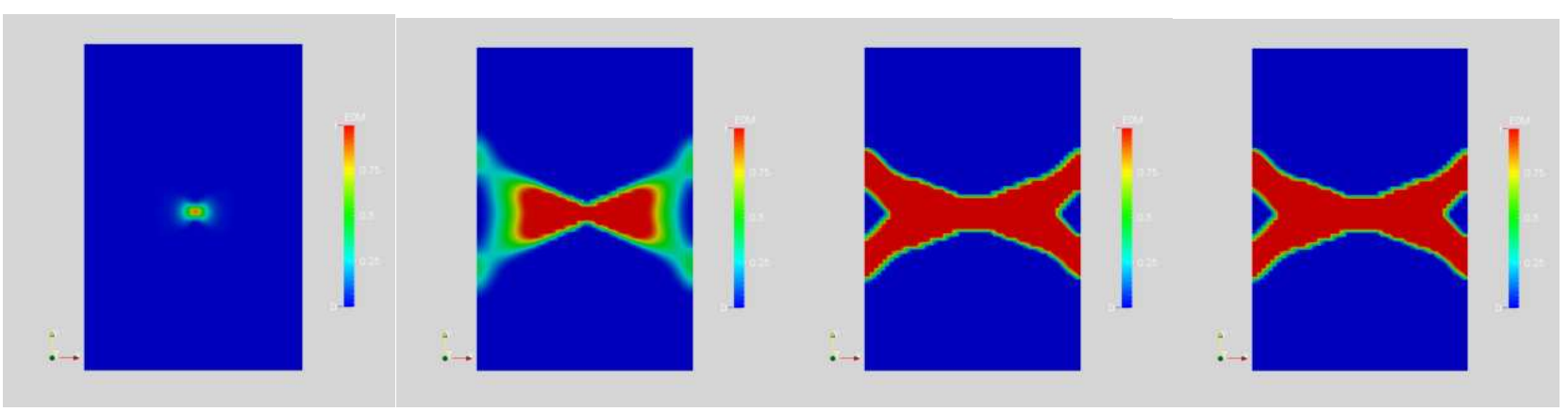

c) Damage field at iterations 50, 62, 100 and 132 of the unstable rupture time step

Figure 14 : Fictive path loading algorithm results for a tensile smooth specimen. 


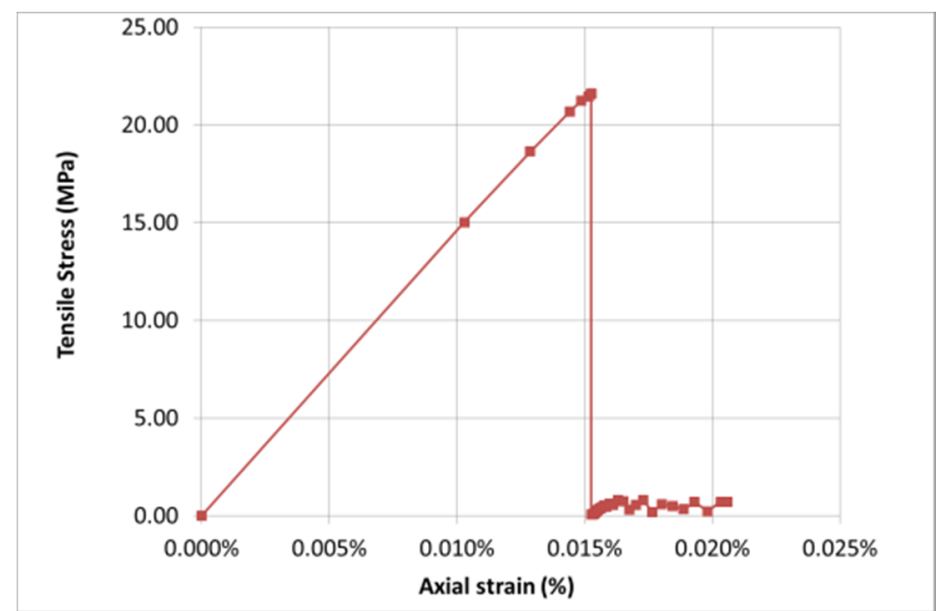

a) Stess-strain curve

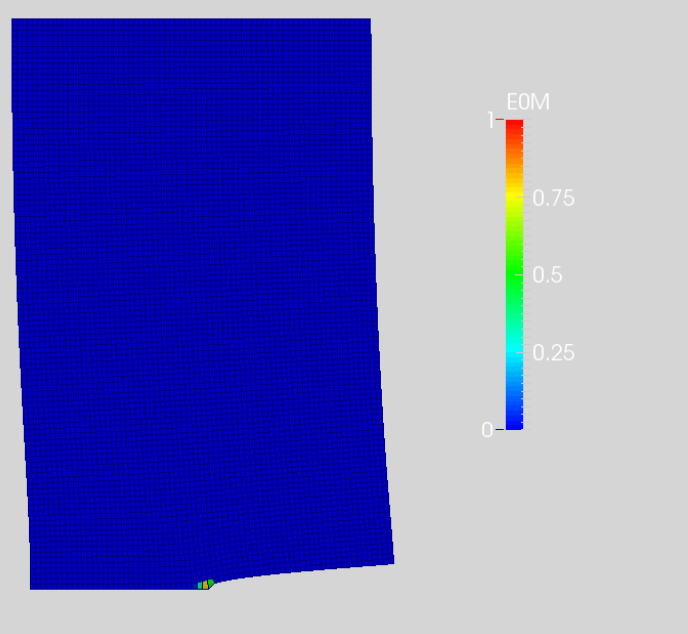

b) Damage field at maximum loading (displacement amplification factor=100)
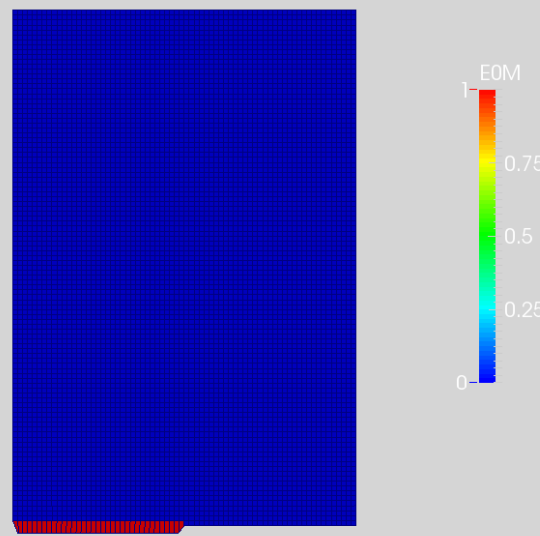

c) Damage field after crack extension (displacement amplification factor=100) Figure 15 : Fictive path loading algorithm results for a tensile cracked specimen. 


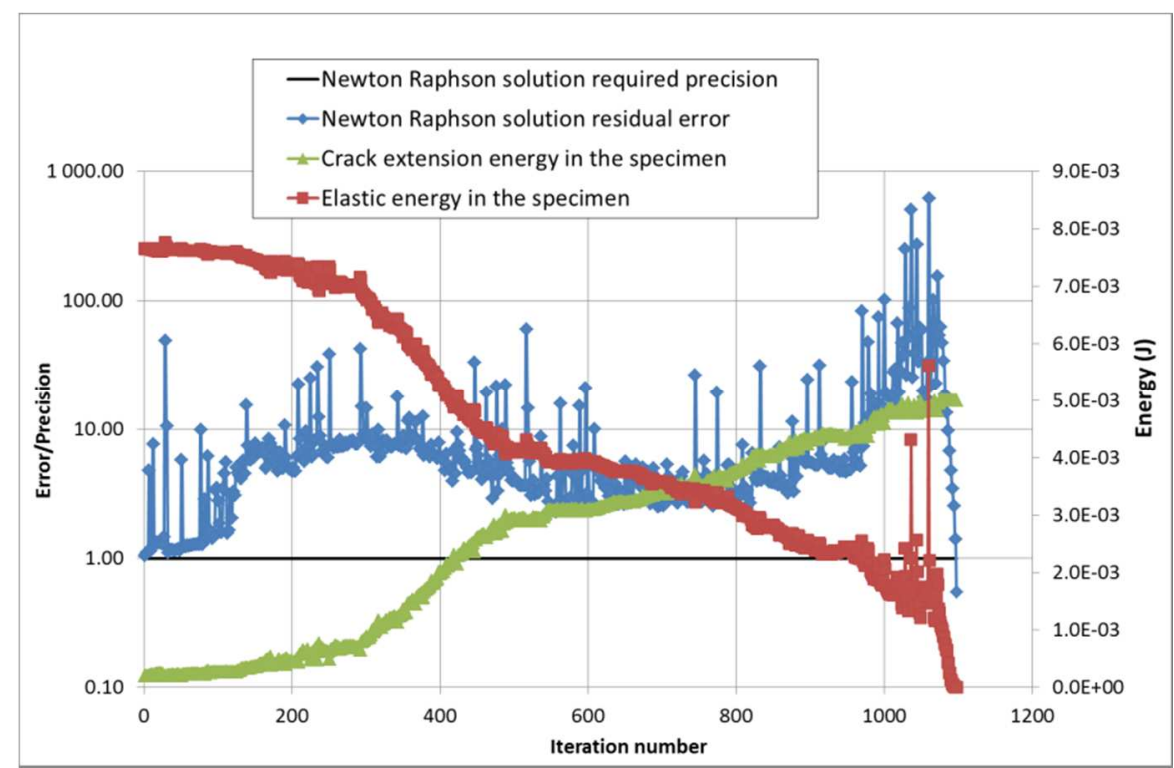

a) Convergence of the fictive path loading algorithm during the rupture time step

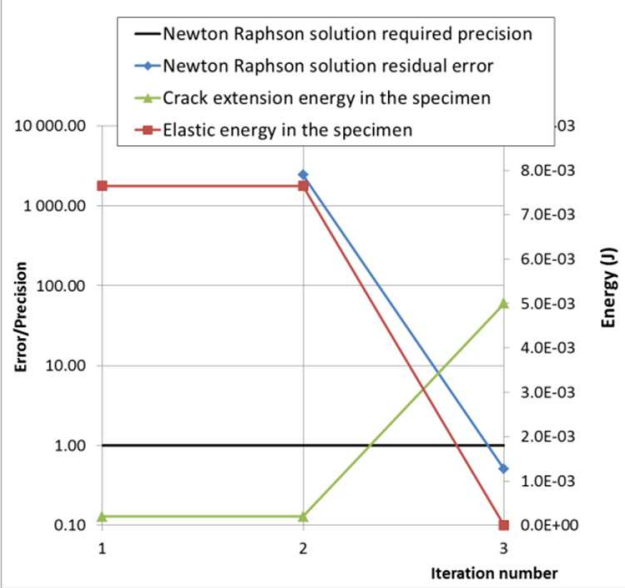

b) Convergence of the quasi Newton algorithm initialized with the fictive path loading solution

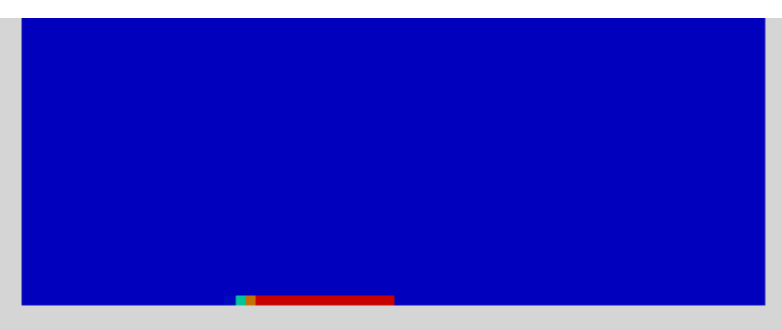

d) Damage extension at iteration 400 of the fictive path loading algorithm

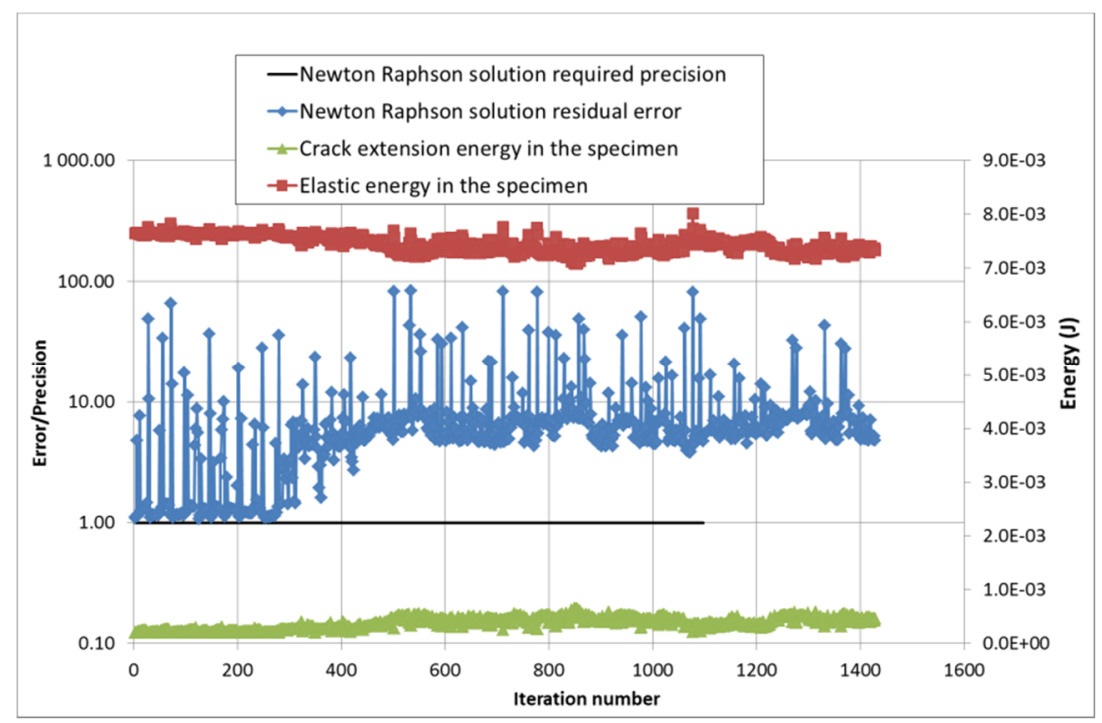

c) Convergence of the quasi Newton algorithm without initialization of the displacement solution Figure 16: Verification of the fictive path loading algorithm solution. 


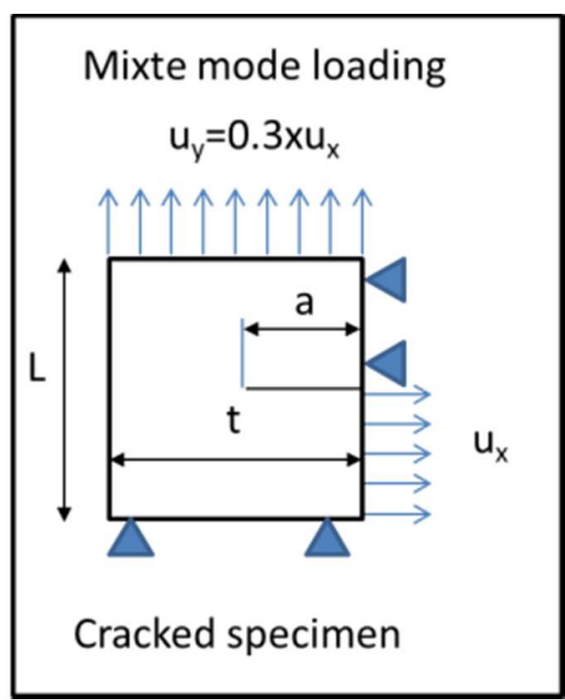

a) Geometry and loading
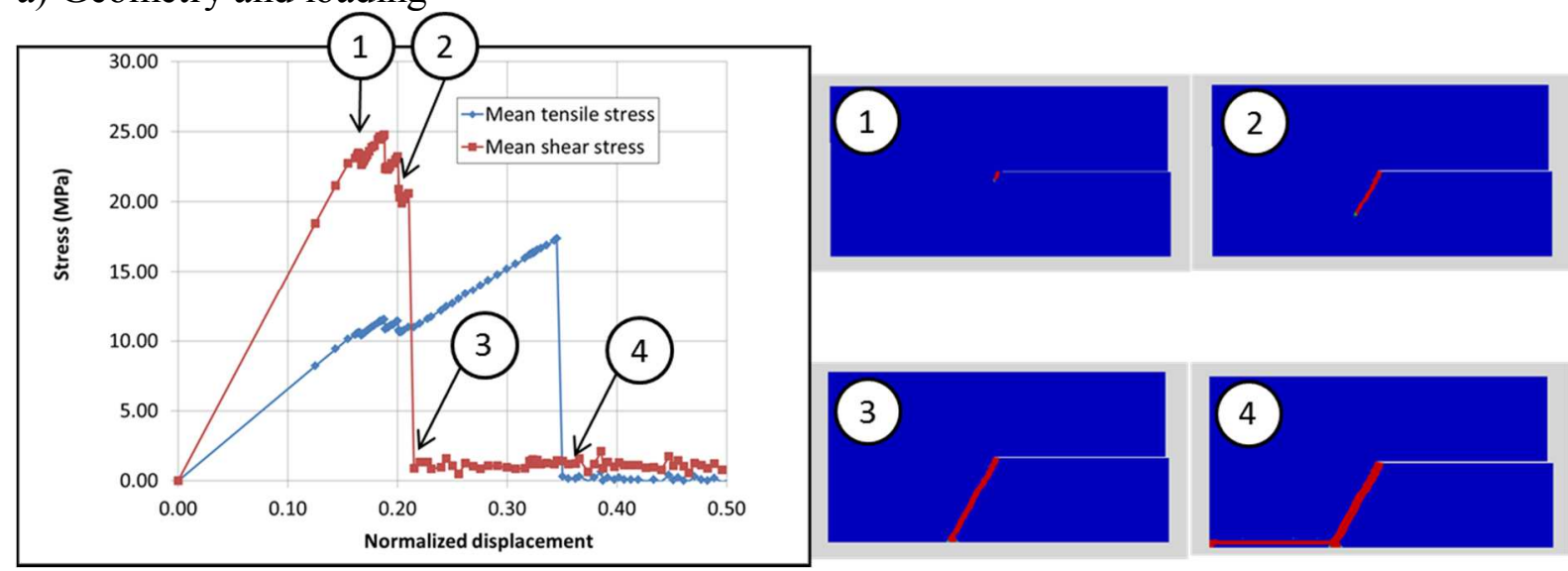

b) Crack extension results

Figure 17 : Unstable crack propagation under mixte mode loading I and II.

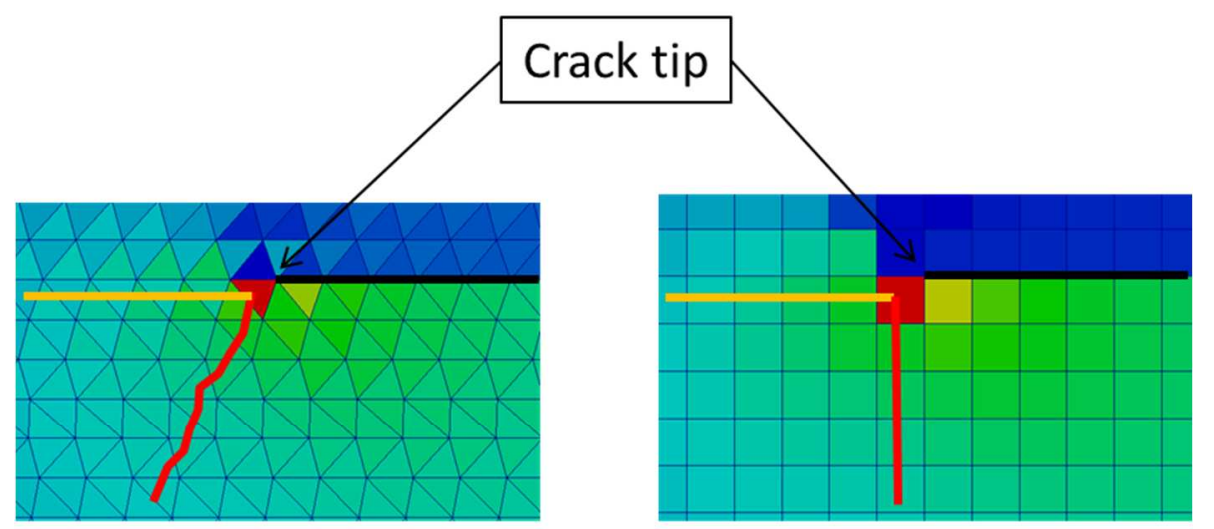

\section{- Mode I crack path \\ - Mode II crack path}

Figure 18 : Impact of the finite element type on crack path. 


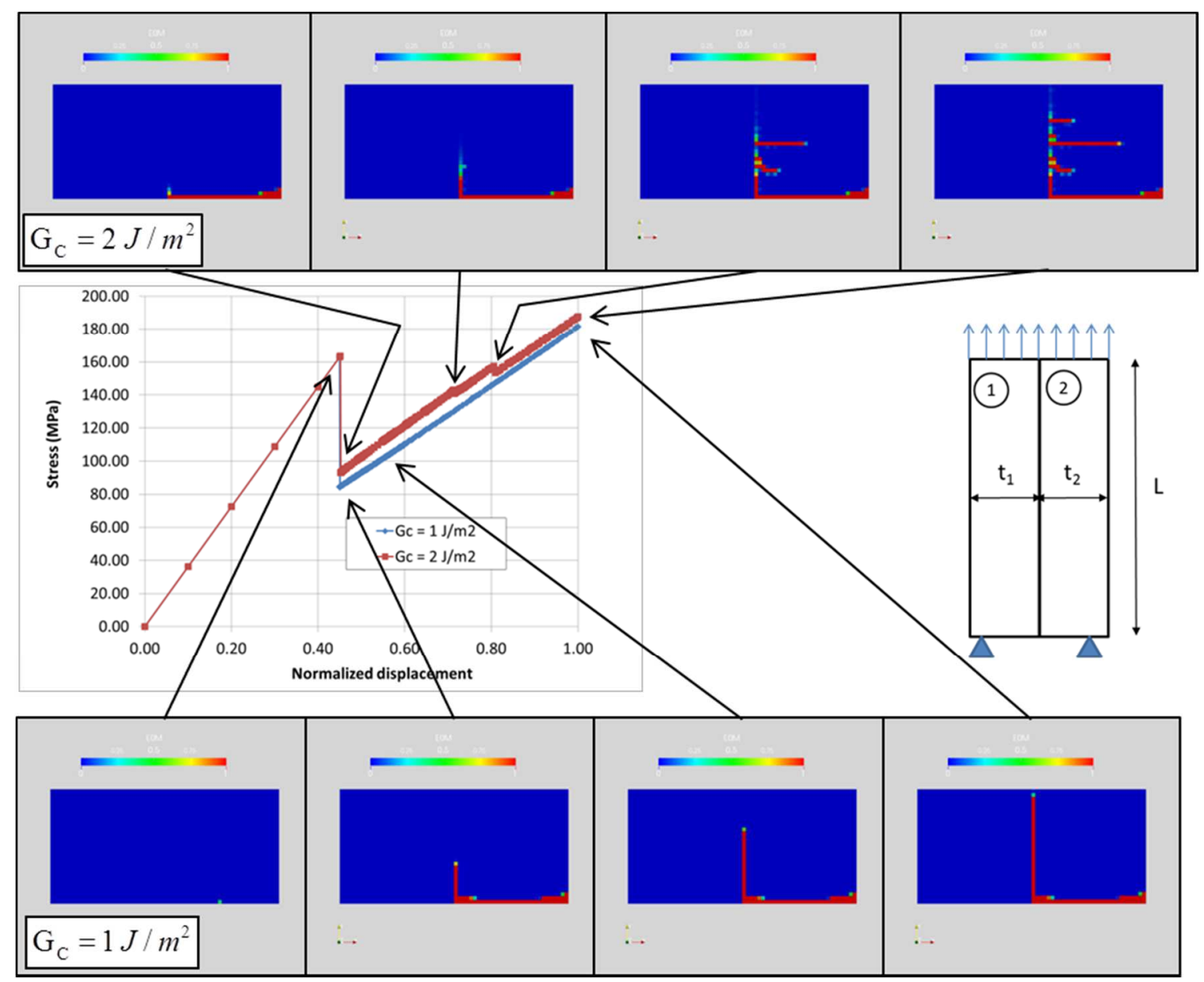

Figure 19 : Crack initiation and growth in a bi-material tensile test specimen.

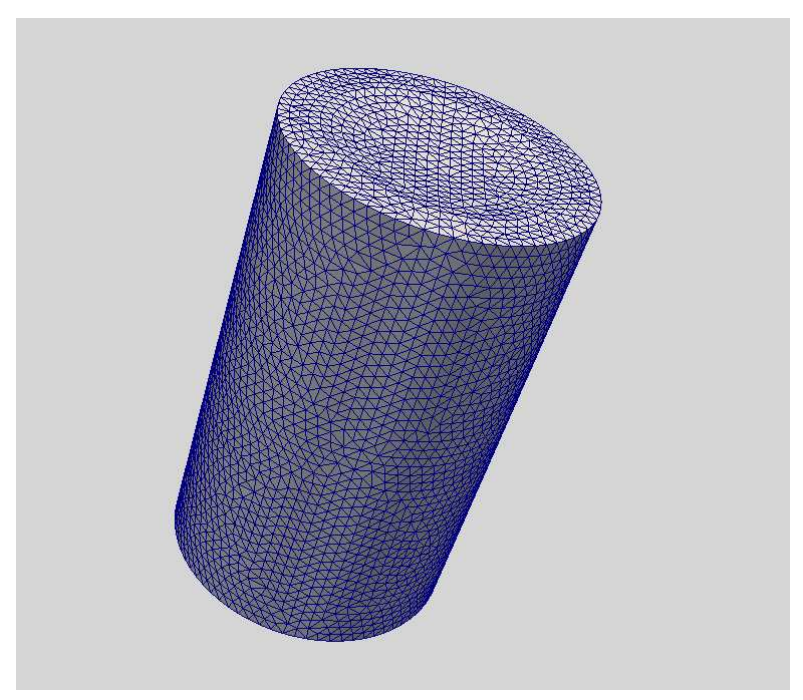

Figure $20: 3 \mathrm{D}$ finite element mesh for nuclear ceramic fragmentation under thermal gradient. 


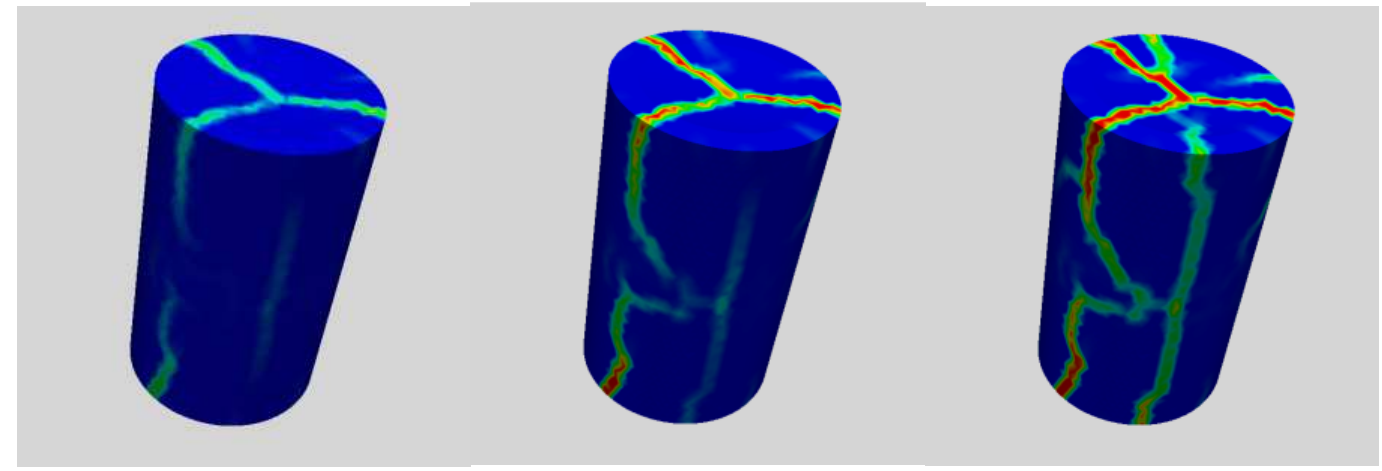

a) $-25{ }^{\circ} \mathrm{C} / \mathrm{mm}$

b) $-50{ }^{\circ} \mathrm{C} / \mathrm{mm}$

c) $-90{ }^{\circ} \mathrm{C} / \mathrm{mm}$
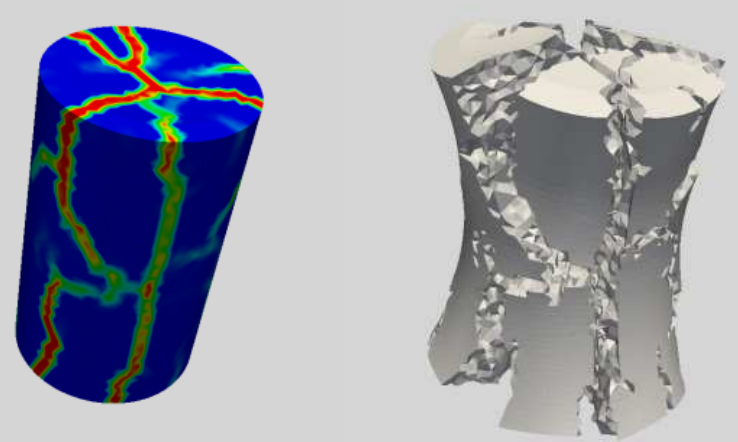

d) $-150^{\circ} \mathrm{C} / \mathrm{mm}$

e) $-150{ }^{\circ} \mathrm{C} / \mathrm{mm}$ (displacement amplification factor $=50$ )

Figure 21 : Numerical results of the nuclear fuel pellet fragmentation simulation for different thermal gradient loading levels

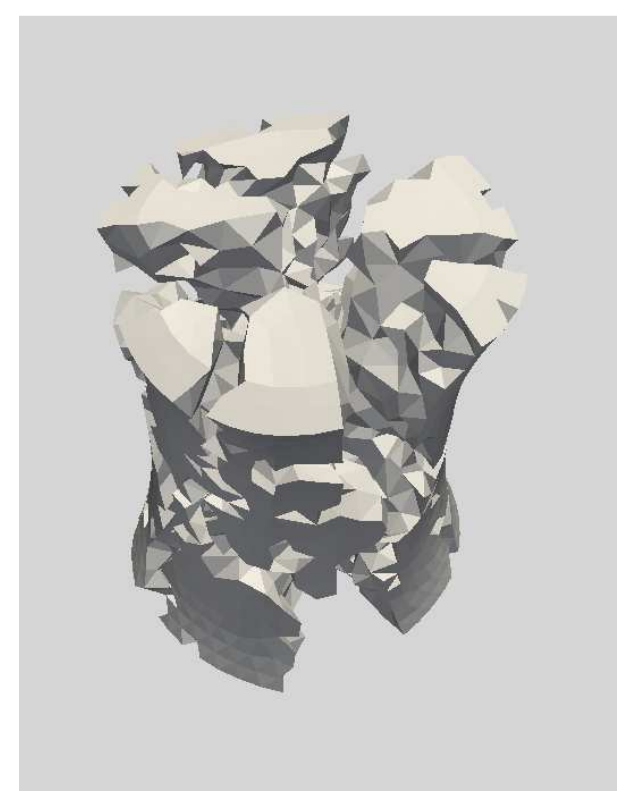

a) Mesh 13340 nodes

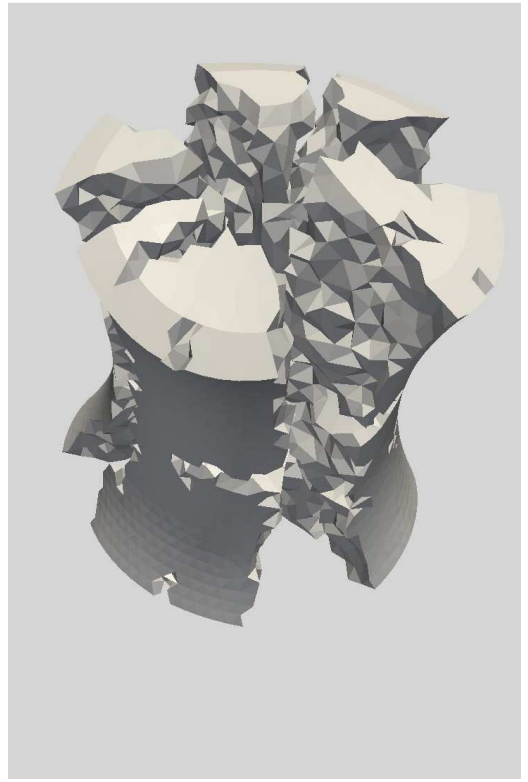

b) Mesh 27658 nodes

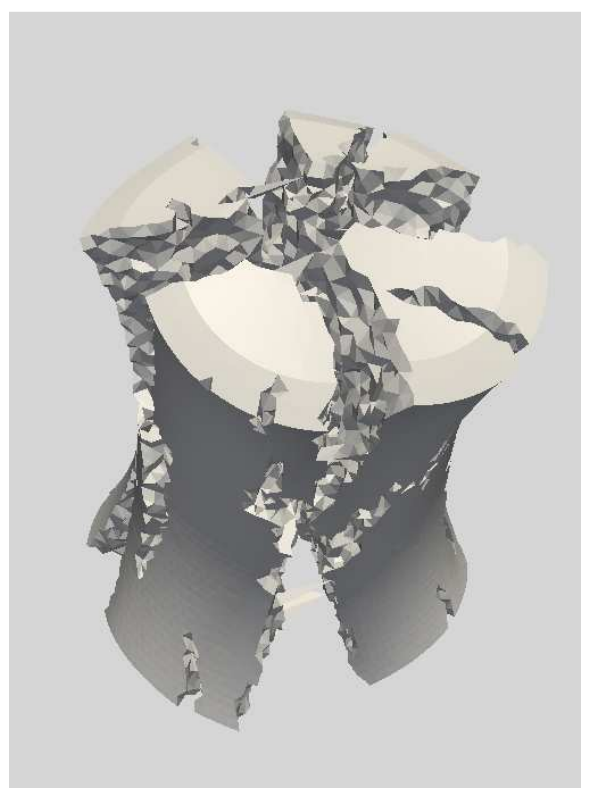

d) Mesh 324397 nodes

Figure 22 : Mesh sensitivity analysis for the nuclear fuel pellet fragmentation (displacement amplification factor $=50$ ) 


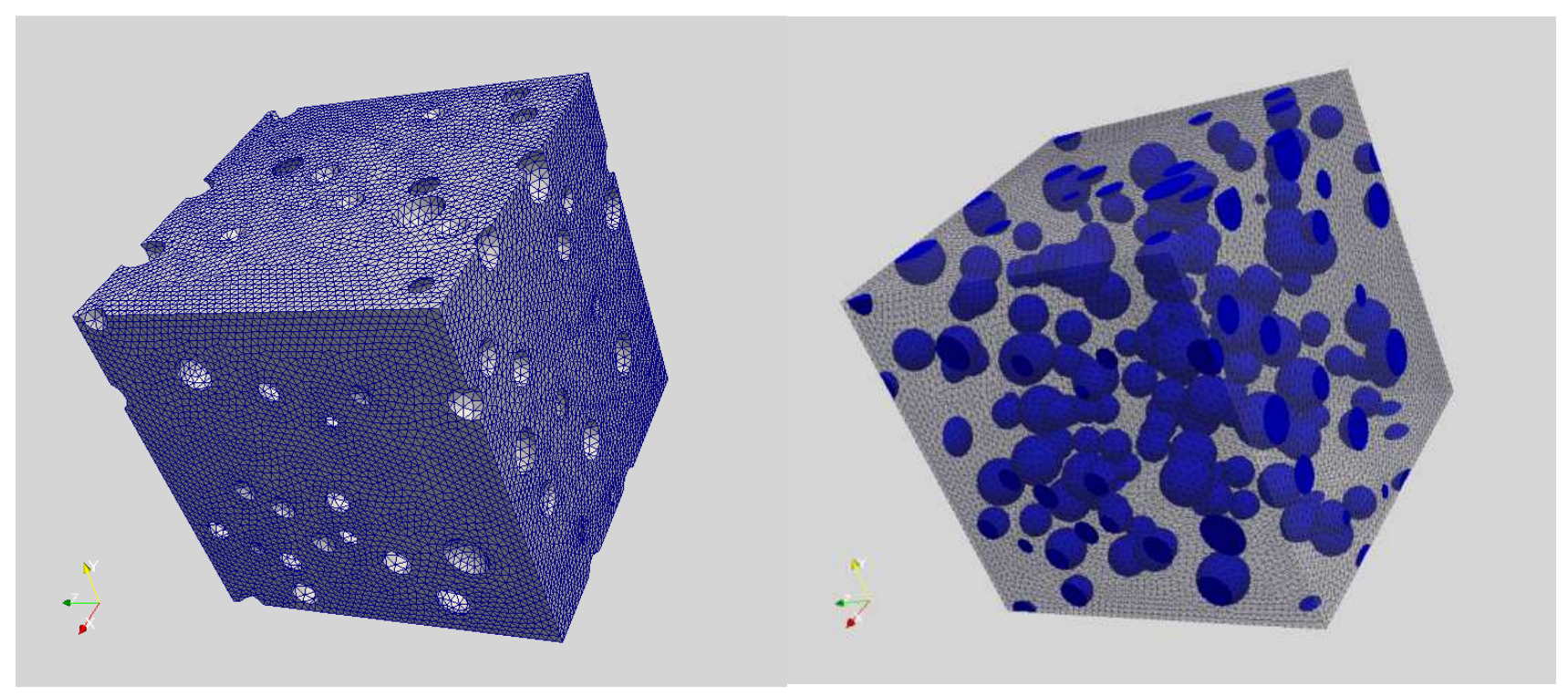

Figure 23 : Finite element mesh for the 3D volume element model of nuclear fuel. 


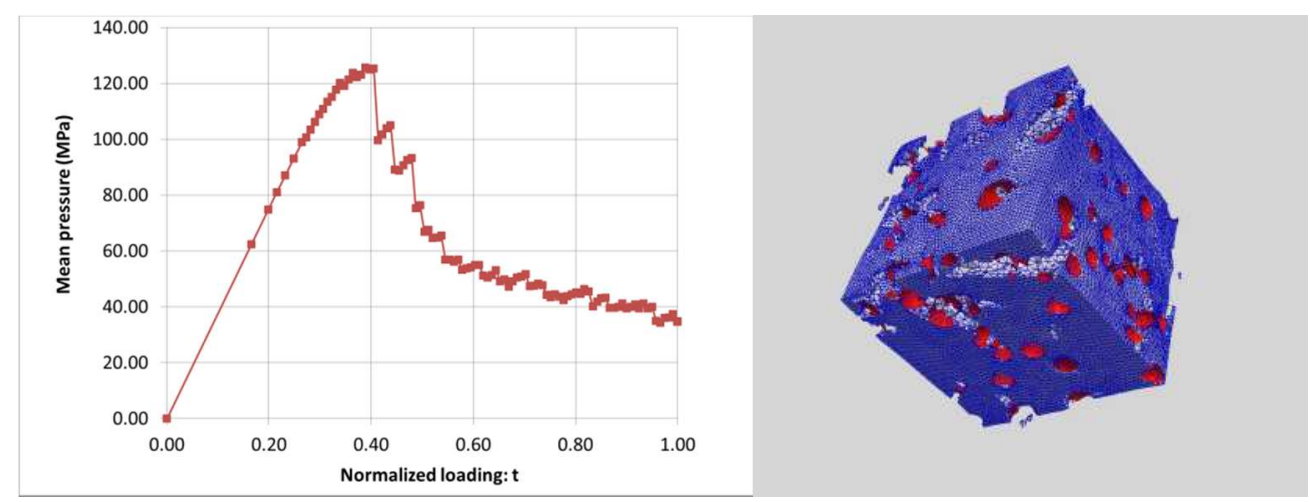

a) Mean pressure in the fuel

b) Pellet fragmentation $\mathrm{t}=0.4$

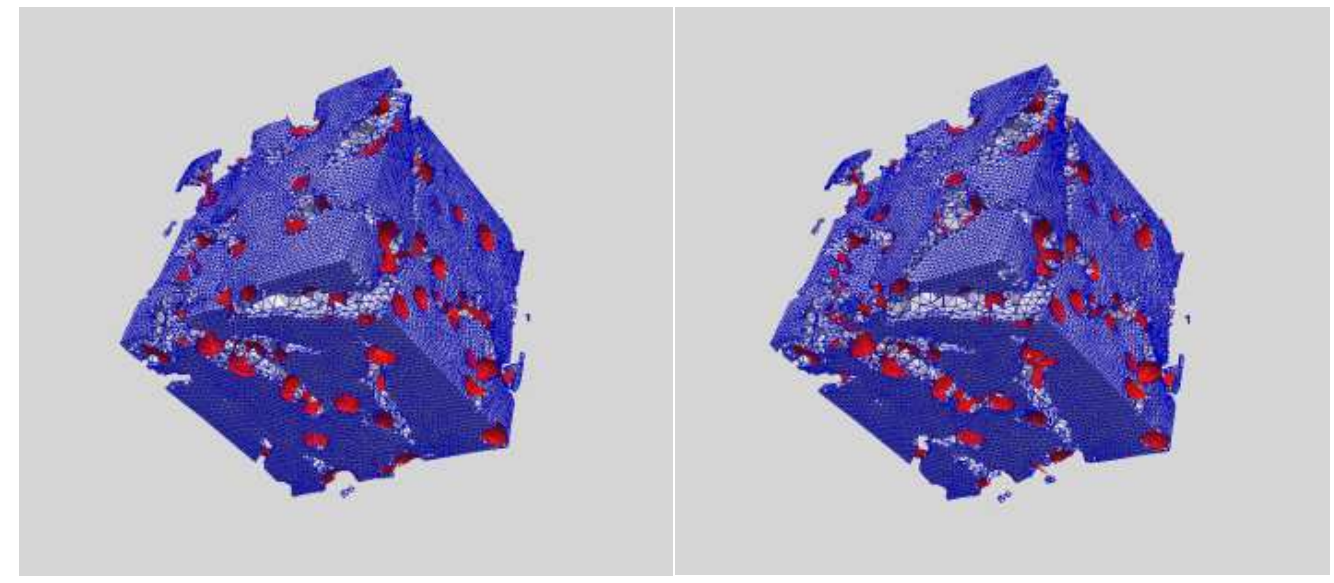

c) Pellet fragmentation $\mathrm{t}=0.44$

d) Pellet fragmentation $\mathrm{t}=0.48$

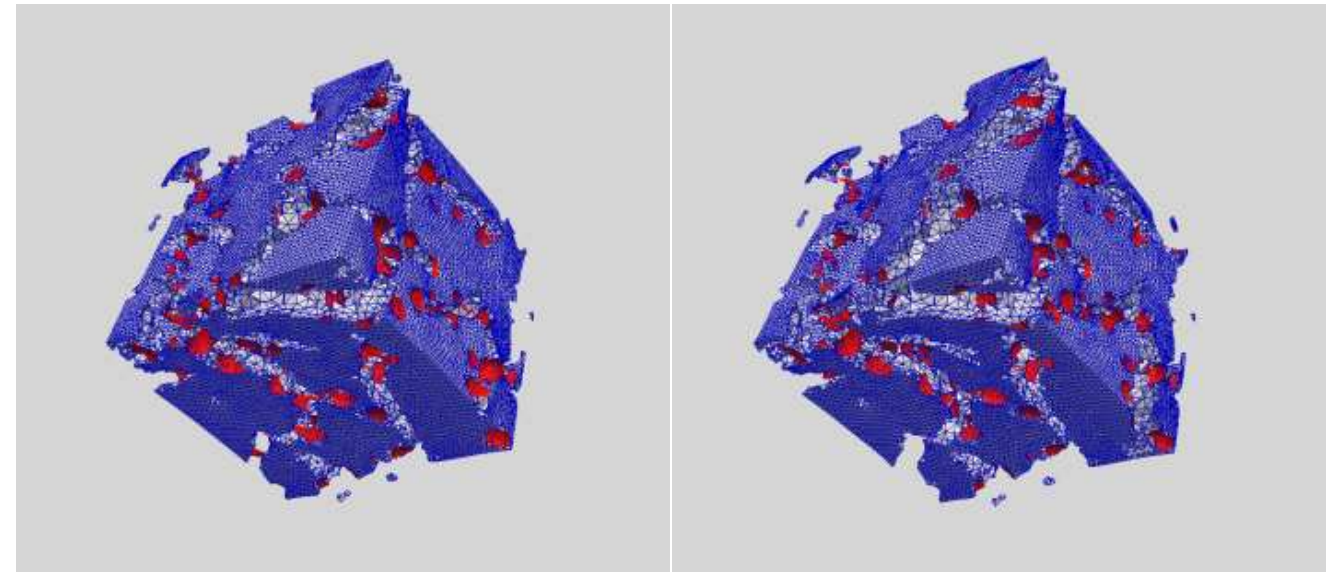

e) Pellet fragmentation $\mathrm{t}=0.5 \quad$ f) Pellet fragmentation $\mathrm{t}=0.54$

Figure 24 : Numerical results for fuel fragmentation at microscopic scale (displacement amplification factor $=500)$. 